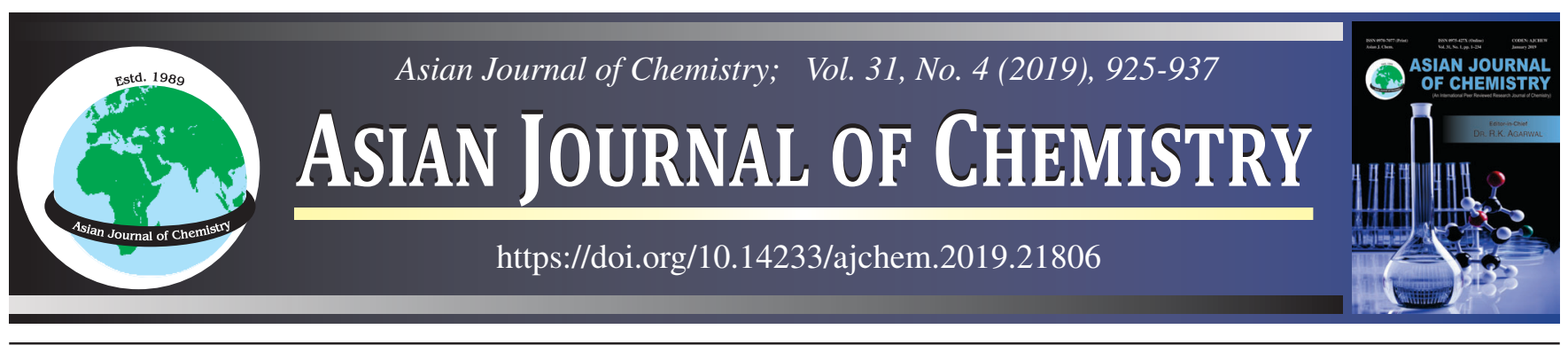

\title{
Applicability of NBO and AIM Topology Analyses to Chemical Bonding in Some Diacetylplatinum(II) Complexes
}

\author{
SAIEd M. Soliman ${ }^{1,2, *}$ and Mohamed M. Zaid ${ }^{1,3, *}$
}

${ }^{1}$ Department of Chemistry, Rabigh College of Science and Art, King Abdulaziz University, P.O. Box 344, Rabigh 21911, Saudi Arabia ${ }^{2}$ Department of Chemistry, Faculty of Science, Alexandria University, P.O. Box 426, Ibrahimia, Alexandria 21321, Egypt

${ }^{3}$ Department of Environmental and Occupational Medicine, National Research Centre, Cairo 12311, Egypt

*Corresponding authors: E-mail: saied1soliman@yahoo.com; mfouad74@yahoo.com

Received: 10 November 2018;

Accepted: 14 January 2019;

Published online: 27 February 2019;

AJC-19308

The geometrical structures of six square-planar diacetylplatinum(II) complexes $\left(\left[\mathrm{Pt}(\mathrm{Ac})_{2} \mathrm{~L}\right], \mathrm{L}=\right.$ hydrazine-type ligand) were calculated using six (B3LYP, CAM-B3LYP, B3PW91, M06, M06HF and PBE) DFT methods and one post-Hartree-Fock (MP2) method combined with 6-31G(d,p) basis sets for nonmetal atoms and LANL2DZ for Pt. Using percent relative errors, M06HF and MP2 are best for predicting Pt-N bond distances, but worst for Pt-C bond distances, whereas B3PW91 is best. Pt-N(pyridine) bonds have higher electron density at the bond critical points than $\mathrm{Pt}-\mathrm{N}$ (hydrazone) bonds, and the former are more covalent than the latter. Further, $\mathrm{Pt}-\mathrm{C}$ bonds trans to hydrazone moieties are more covalent than $\mathrm{Pt}-\mathrm{C}$ bonds trans to $\mathrm{Pt}-\mathrm{N}$ (pyridine) bonds. $\mathrm{Pt}-\mathrm{C}$ bonds are mainly due to $\mathrm{Pt} \rightarrow \mathrm{Ac}$ back donation rather than $\mathrm{Ac} \rightarrow \mathrm{Pt}$ donation; $\sigma$-bonding is less important in this case, consistent with the high $\pi$-acidity and strong trans effect of acetyl groups. In contrast, $\mathrm{Pt} \rightarrow \mathrm{N}$ back donation is negligible and $\mathrm{Pt}-\mathrm{N}$ bonds are mainly due to $\mathrm{N} \rightarrow \mathrm{Pt} \sigma$-donation, which stabilizes trans $\mathrm{Pt}-\mathrm{C}$ bonds.

Keywords: Pt-C bonds, Pt-N bonds, B3PW91, NBO, AIM, Topology analysis, Acetyl ligands.

ᄂ - - - - - - - - - - - - - - - - - - - - - - - - - - - -

\section{INTRODUCTION}

Platinum(II) complexes are considered the mainstay of clinical chemotherapy regimens. Cisplatin [1] is the corner stone for Pt-based anticancer drugs, and it is used as chemotherapy for the treatment of various cancers [2,3]. Currently, cisplatin and its analogue carboplatin are used for treating ovarian and testicular cancers, which have high healing rates. Owing to the interesting biological effects of platinum compounds, many Pt complexes have been synthesized and used as anticancer drugs [4-9]. In vitro studies have indicated that many Pt(II) complexes [6,9-12] have higher efficiencies than cisplatin as anticancer drugs.

It is believed that the mechanism of action of Pt-based drugs, such as cisplatin, against cancer includes aquation (chloride replacement by water) of the complex, followed by interaction with DNA purine bases. These steps are strongly related to the stability and nature of bonding around platinum, i.e., Pt-ligand bonds [13]. From this point of view, under- standing the nature of the bonding environment around $\mathrm{Pt}^{2+}$ is of great importance.

Criteria for identifying and studying intermolecular interactions, such as metal-ligand bonding, by the atoms in molecules (AIM) theory have been proposed by Popelier and Bader $[14,15]$. Bader's AIM theory [16] provides important information about the nature of bonding environments. Bonds can be characterized by following many topological properties at the $(3,-1)$ bond critical points (BCPs). The BCPs are points along the bond path between two bonded atoms where the electron density reaches a local minimum. Topological properties, such as total electron density, $\rho(r)$, and the laplacian of electron density, $\nabla^{2} \rho(r)$, at various BCPs, give an indication of the nature of bonding and non-bonding interactions among atoms in a molecule. Moreover, natural bond orbital (NBO) analysis is another significant route for studying interactions among atoms in molecular systems.

Here, we have demonstrated the efficiency of some DFT methods for predicting the molecular structure, specially, the

This is an open access journal, and articles are distributed under the terms of the Creative Commons Attribution-NonCommercial-ShareAlike 4.0 (CC BY-NC-SA 4.0) International License which allows readers to freely read, download, copy, distribute, print, search, or link to the full texts of its articles and to use them for any other lawful non-commercial purpose as long as the original source is duly acknowledged. 
metal-ligand bonds, of some recently synthesized hydrazinediacetyl platinum(II) complexes; $\left[\mathrm{Pt}(\mathrm{Ac})_{2} \mathrm{~L}\right]$ as shown in Fig. 1. Six (B3LYP, CAM-B3LYP, B3PW91, M06, M06HF and PBE) DFT methods were used in this work. In addition, the results were compared with those obtained using the postHartree-Fock $a b$ intio MP2 method. We tested the efficiency of these functionals for predicting $\mathrm{Pt}-\mathrm{N}$ and $\mathrm{Pt}-\mathrm{C}$ bond distances, as compared to experimental X-ray structure results. Moreover, we used AIM and NBO analyses to describe the nature of the $\mathrm{Pt}-\mathrm{N}$ and $\mathrm{Pt}-\mathrm{C}$ bonds [17].

\section{COMPUTATIONAL METHODS}

The Gaussian 09 and Gauss View programs [18] were used to optimize the molecular structures of six Pt complexes as shown in Fig. 1 using different levels of theory. In this study, we used six DFT functionals, namely, B3LYP [19,20], CAMB3LYP [21], B3PW91 [22], M06 [23], M06HF [23] and PBE [24]. The basis sets used were 6-31G(d,p) [25] for nonmetal atoms (C, H, N, O and F) and LANL2DZ for Pt [26]. Using effective core potentials (ECPs) is important in reducing computational expense and is considered adequate for geometry optimization and bond properties in metal-organic complexes, specially for heavy element such as platinum [27]. Following geometry optimization, frequency calculations were carried out at the optimized structures. All gave real frequencies and no imaginary vibrational modes were detected. Further, the geometries were optimized using the post-Hartree-Fock $a b$ initio MP2 method [28]. This method has a higher computational cost than DFT methods, but is believed to give accurate predictions of molecular structure characteristics. In this case, the Density=Current keyword is used in order to include the post Hartree-Fock density in NBO analysis and wavefunction files. At the optimized structures, single-point calculations were carried out to create wave function files, which contain all the data needed for the AIM analyses. The Multiwfn program [29] was used to run the wave function files for topology analysis of the studied complexes. In addition, natural population analyses were conducted using the NBO 3.1 program package [30], which is built in the Gaussian 09 software. Similarly, singlepoint calculations at the X-ray structures were performed for all the studied complexes, followed by AIM and NBO analyses at the experimental geometries. The starting input structures were extracted from crystallographic information files (CIFs) obtained from the Cambridge Crystallographic Database Centre (CCDC Nos. 95819-95823 and 95825) [17].

\section{RESULTS AND DISCUSSION}

Methods assessment: The MP2 optimized geometries and atom-numbering schemes of the studied $\mathrm{Pt}$ complexes are shown in Fig. 1. The calculated Pt-N/Pt-C distances obtained by quantum chemical geometry optimizations of the studied $\mathrm{Pt}$ complexes using different methods are compared with Xray results in Table-1. For all data, the percent relative error (\% err) values are shown in Table-2. As can be seen from these tables, all the methods overestimate the Pt-N bond distances, but the M06HF and MP2 methods are the best for predicting $\mathrm{Pt}-\mathrm{N}$ distances. Surprisingly, the minimum absolute relative errors (\% err.) for the Pt-N bond distances obtained by the M06HF method were very close to those obtained using the MP2 method, which has a higher computational cost. In contrast, the M06HF and MP2 methods have the worst accuracy for predicting the $\mathrm{Pt}-\mathrm{C}$ bond distances, showing the highest

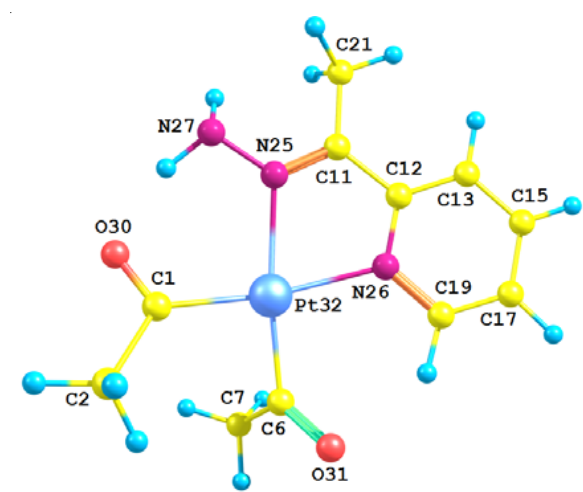

Complex 1

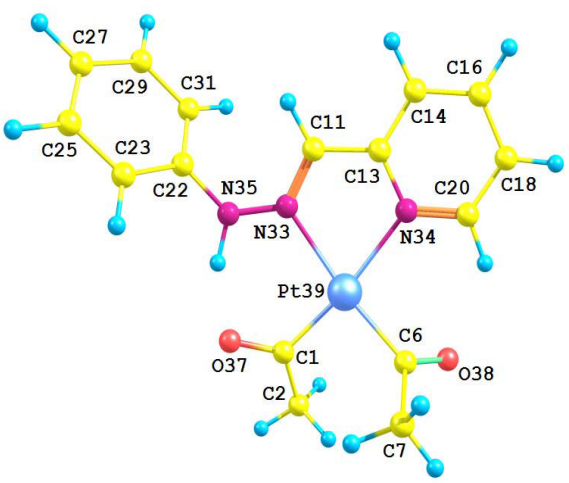

Complex 4

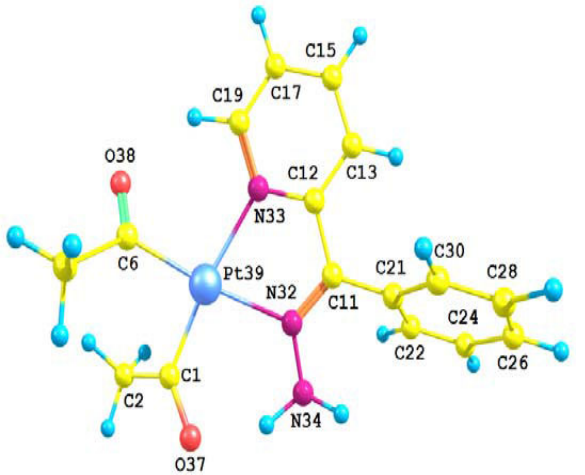

Complex 2

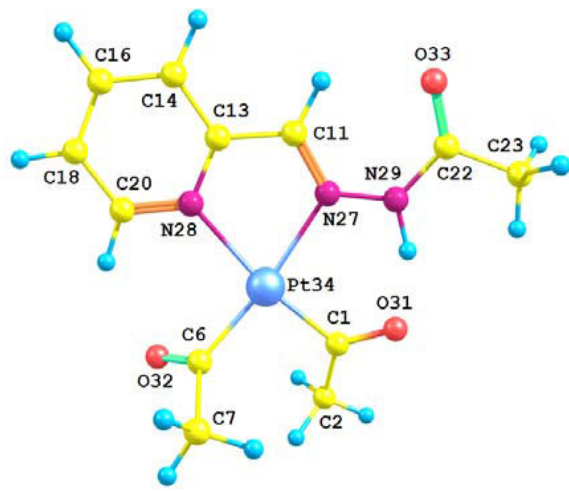

Complex 5
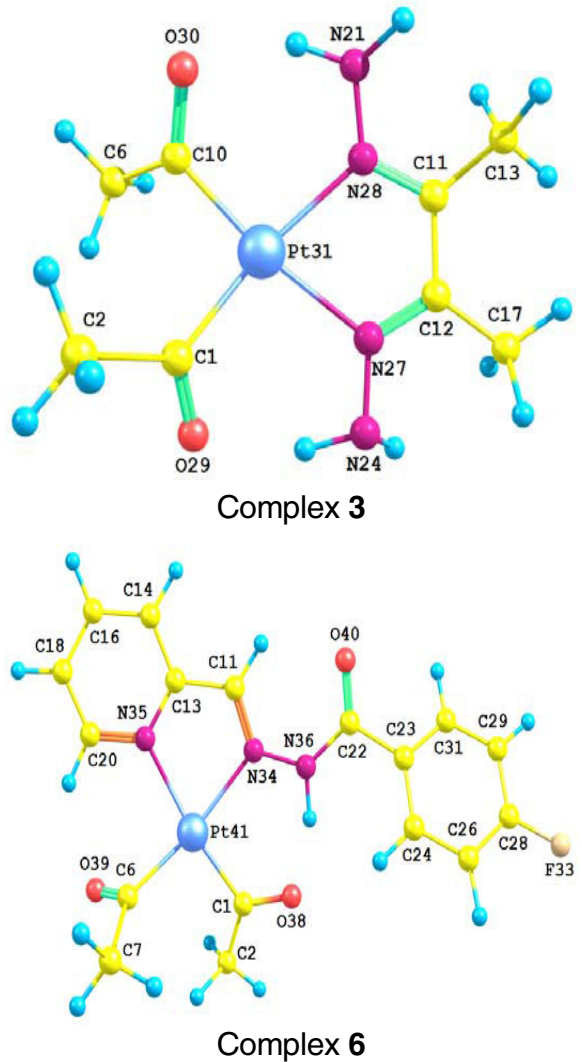

Fig. 1. MP2 optimized structures and atom-numbering schemes of the studied Pt complexes 


\begin{tabular}{|c|c|c|c|c|c|c|c|c|}
\hline \multicolumn{9}{|c|}{$\begin{array}{r}\text { TABLE-1 } \\
\text { CALCULATED AND EXPERIMENTAL Pt-N AND Pt-C BOND }\end{array}$} \\
\hline & B3LYP & B3PW91 & CAM-B3LYP & M06 & M06HF & PBE & MP2 & Exp. [Ref. 17] \\
\hline \multicolumn{9}{|c|}{ Complex 1} \\
\hline Pt32-N25 & 2.2358 & 2.2004 & 2.2015 & 2.2482 & 2.1822 & 2.2108 & 2.1772 & 2.1311 \\
\hline Pt32-N26 & 2.2167 & 2.1843 & 2.1878 & 2.2285 & 2.1766 & 2.1977 & 2.1735 & 2.1267 \\
\hline Pt32-C1 & 2.0081 & 1.9942 & 1.9981 & 2.0104 & 1.9508 & 2.0003 & 1.9563 & 2.0044 \\
\hline Pt32-C6 & 2.0023 & 1.9896 & 1.9905 & 2.0013 & 1.9504 & 1.9975 & 1.9583 & 1.9884 \\
\hline \multicolumn{9}{|c|}{ Complex $2^{a}$} \\
\hline Pt39-N33 & 2.2447 & 2.2085 & 2.2096 & 2.2626 & 2.1979 & 2.2199 & 2.1793 & 2.1458 \\
\hline Pt39-N34 & 2.2166 & 2.1849 & 2.1873 & 2.2224 & 2.1691 & 2.1972 & 2.1740 & 2.1138 \\
\hline Pt39-C1 & 2.0083 & 1.9943 & 1.9984 & 2.0126 & 1.9548 & 2.0005 & 1.9569 & 1.9805 \\
\hline Pt39-C6 & 2.0019 & 1.9891 & 1.9899 & 1.9982 & 1.9472 & 1.9964 & 1.9586 & 1.9783 \\
\hline \multicolumn{9}{|c|}{ Complex 3} \\
\hline Pt31-N27 & 2.2178 & 2.1836 & 2.1867 & 2.2233 & 2.1775 & 2.1979 & 2.1566 & 2.1314 \\
\hline Pt31-N28 & 2.2177 & 2.1835 & 2.1868 & 2.2231 & 2.1774 & 2.1980 & 2.1565 & 2.1282 \\
\hline Pt31-C1 & 2.0050 & 1.9912 & 1.9948 & 2.0066 & 1.9503 & 1.9975 & 1.9566 & 1.9936 \\
\hline Pt31-C10 & 2.0050 & 1.9912 & 1.9949 & 2.0066 & 1.9503 & 1.9975 & 1.9565 & 1.9994 \\
\hline \multicolumn{9}{|c|}{ Complex 4} \\
\hline Pt39-N33 & 2.2799 & 2.2383 & 2.2373 & 2.2922 & 2.2011 & 2.2558 & 2.1916 & 2.1599 \\
\hline Pt39-N34 & 2.2181 & 2.1849 & 2.1904 & 2.2263 & 2.1688 & 2.1981 & 2.1698 & 2.1191 \\
\hline Pt39-C1 & 2.0059 & 1.9922 & 1.9974 & 2.0114 & 1.9536 & 1.9974 & 1.9564 & 1.9938 \\
\hline Pt39-C6 & 1.9975 & 1.9853 & 1.9862 & 1.9959 & 1.9467 & 1.9925 & 1.9564 & 1.9850 \\
\hline \multicolumn{9}{|c|}{ Complex $5^{\mathrm{a}}$} \\
\hline Pt34-N27 & 2.2735 & 2.2304 & 2.2329 & 2.2855 & 2.2019 & 2.2401 & 2.2036 & 2.1683 \\
\hline Pt34-N28 & 2.2280 & 2.1905 & 2.1957 & 2.2322 & 2.1734 & 2.2047 & 2.1716 & 2.1315 \\
\hline $\mathrm{Pt} 34-\mathrm{C} 1$ & 2.0070 & 1.9929 & 1.9980 & 2.0128 & 1.9552 & 1.9993 & 1.9566 & 1.9806 \\
\hline Pt34-C6 & 1.9977 & 1.9865 & 1.9866 & 1.9966 & 1.9469 & 1.9953 & 1.9539 & 1.9772 \\
\hline \multicolumn{9}{|c|}{ Complex 6} \\
\hline Pt41-N34 & 2.2786 & 2.2399 & 2.2394 & 2.2921 & 2.2061 & 2.2532 & 2.2078 & 2.1694 \\
\hline Pt41-N35 & 2.2210 & 2.1861 & 2.1924 & 2.2275 & 2.1646 & 2.2003 & 2.1696 & 2.1225 \\
\hline $\mathrm{Pt} 41-\mathrm{C} 1$ & 2.0048 & 1.9903 & 1.9962 & 2.0112 & 1.9536 & 1.9966 & 1.9533 & 1.9799 \\
\hline Pt41-C6 & 1.9983 & 1.9860 & 1.9866 & 1.9968 & 1.9470 & 1.9943 & 1.9538 & 1.9770 \\
\hline
\end{tabular}

${ }^{\mathrm{a}}$ Experimental bond distances are the average of the two units observed in the crystal.

TABLE-2

THE CALCULATED PERCENT RELATIVE ERRORS (\% err.) OF THE CALCULATED Pt-N AND Pt-C BOND DISTANCES

\begin{tabular}{|c|c|c|c|c|c|c|c|}
\hline Bond & B3LYP & B3PW91 & CAM-B3LYP & M06 & M06HF & PBE & MP2 \\
\hline \multicolumn{8}{|c|}{ Complex 1} \\
\hline Pt-N25 & 4.911 & 3.248 & 3.299 & 5.494 & 2.396 & 3.738 & 2.159 \\
\hline Pt-N26 & 4.236 & 2.711 & 2.877 & 4.790 & 2.348 & 3.340 & 2.203 \\
\hline $\mathrm{Pt}-\mathrm{C} 1$ & 0.188 & -0.509 & -0.312 & 0.300 & -2.675 & -0.205 & -2.398 \\
\hline Pt-C6 & 0.700 & 0.059 & 0.106 & 0.651 & -1.910 & 0.457 & -1.513 \\
\hline \multicolumn{8}{|c|}{ Complex 2} \\
\hline Pt-N33 & 4.609 & 2.920 & 2.972 & 5.442 & 2.427 & 3.455 & 1.559 \\
\hline Pt-N34 & 4.866 & 3.366 & 3.480 & 5.138 & 2.616 & 3.945 & 2.848 \\
\hline Pt-C1 & 1.403 & 0.698 & 0.904 & 1.621 & -1.300 & 1.010 & -1.195 \\
\hline Pt-C6 & 1.194 & 0.546 & 0.588 & 1.004 & -1.570 & 0.917 & -0.994 \\
\hline \multicolumn{8}{|c|}{ Complex 3} \\
\hline Pt-N27 & 4.050 & 2.448 & 2.593 & 4.309 & 2.161 & 3.120 & 1.182 \\
\hline Pt-N28 & 4.204 & 2.598 & 2.753 & 4.459 & 2.312 & 3.277 & 1.328 \\
\hline $\mathrm{Pt}-\mathrm{C} 1$ & 0.570 & -0.123 & 0.061 & 0.651 & -2.172 & 0.193 & -1.854 \\
\hline $\mathrm{Pt}-\mathrm{C} 10$ & 0.280 & -0.412 & -0.226 & 0.359 & -2.456 & -0.096 & -2.144 \\
\hline \multicolumn{8}{|c|}{ Complex 4} \\
\hline Pt-N33 & 5.553 & 3.629 & 3.581 & 6.124 & 1.906 & 4.438 & 1.470 \\
\hline Pt-N34 & 4.676 & 3.105 & 3.368 & 5.062 & 2.346 & 3.730 & 2.395 \\
\hline Pt-C1 & 0.610 & -0.081 & 0.184 & 0.883 & -2.014 & 0.184 & -1.877 \\
\hline Pt-C6 & 0.629 & 0.013 & 0.061 & 0.547 & -1.928 & 0.377 & -1.440 \\
\hline \multicolumn{8}{|c|}{ Complex 5} \\
\hline Pt-N27 & 4.854 & 2.867 & 2.978 & 5.408 & 1.551 & 3.313 & 1.629 \\
\hline Pt-N28 & 4.530 & 2.768 & 3.014 & 4.725 & 1.968 & 3.438 & 1.881 \\
\hline Pt-C1 & 1.335 & 0.622 & 0.882 & 1.629 & -1.281 & 0.947 & -1.212 \\
\hline Pt-C6 & 1.038 & 0.473 & 0.475 & 0.983 & -1.530 & 0.914 & -1.179 \\
\hline
\end{tabular}




\begin{tabular}{cccccccc}
\hline \multicolumn{7}{c}{ Complex 6 } \\
\hline Pt-N34 & 5.034 & 3.248 & 3.226 & 5.657 & 1.693 & 3.864 & 1.771 \\
Pt-N35 & 4.640 & 2.995 & 3.293 & 4.946 & 1.984 & 3.665 & 2.220 \\
Pt-C1 & 1.259 & 0.525 & 0.827 & 1.580 & -1.324 & 0.846 & -1.343 \\
Pt-C6 & 1.077 & 0.456 & 0.487 & 0.998 & -1.518 & 0.872 & -1.174 \\
\hline
\end{tabular}

percent relative errors among the examined methods. It is clear that both these methods highly under estimated the Pt-C bond distances, whereas most of the other methods slightly over estimated the experimental bond distances. Generally, the percent relative errors for the $\mathrm{Pt}-\mathrm{C}$ bonds are less than those for the Pt-N bonds. For the majority of Pt-C bonds, the B3PW91 method exhibited the best performance. Of the twelve Pt-C bonds, only three (two Pt-C bonds of complex 3 and one Pt-C bond of complex 1) were not well predicted using the B3PW91 method.

For further and accurate inspection of the studied quantum chemical methods, we used, for the first time, AIM topological parameters to assess the methods used for geometry optimization. The total electron density $[\rho(r)]$ at the BCP is a sensitive topological parameter for bond strength that indicates the strength of the interaction between the atoms forming the bond. The $\rho(\mathrm{r})$ values at the $\mathrm{Pt}-\mathrm{N}$ and $\mathrm{Pt}-\mathrm{C}, \mathrm{BCPs}$ are listed in Table-3. The $\rho(\mathrm{r})$ values were computed at the optimized and experimental structures using different methods. For Pt-N bonds, the $\rho(r)$ values at the calculated and experimental struc- tures are most similar with the MP2 and M06HF methods. In contrast, the B3PW91 method gave the best agreement between the calculated and experimental $\rho(\mathrm{r})$ values for $\mathrm{Pt}-\mathrm{C}$ bonds. It is clear from this AIM analysis that both the MP2 and M06HF methods are the best performing for Pt-N bonds, whereas the B3PW91 method is the most accurate for the majority of Pt-C bonds.

AIM study: The nature of the Pt-N and $\mathrm{Pt}-\mathrm{C}$ bonds was investigated using AIM topological parameters. For this purpose, we employed the total electron density $[\rho(\mathrm{r})]$, its laplacian $\left(\nabla^{2} \rho(\mathrm{r})\right)$, the total energy density $[\mathrm{H}(\mathrm{r})]$ and the ratio of the potential energy density to the kinetic energy density $(\mathrm{IV}(\mathrm{r}) / \mathrm{G}(\mathrm{r}))$ at the BCP. The computed topology parameters, $\rho(\mathrm{r}), \mathrm{H}(\mathrm{r}), \mathrm{IV}(\mathrm{r}) / / \mathrm{G}(\mathrm{r})$ and $\nabla^{2} \rho(\mathrm{r})$, at the optimized and X-ray structures are summarized in Tables 3-6.

Bader and Essen [16] observed that shared (covalent) interactions have high $\rho(\mathrm{r})$ values, whereas these values are small for ionic systems and other closed-shell interactions. Table-3 shows that $\rho(\mathrm{r})$ at the $\mathrm{Pt}-\mathrm{N}$ and $\mathrm{Pt}-\mathrm{C}, \mathrm{BCPs}$ are in the range of 0.0653-0.0862 a.u. (exp. 0.0815-0.0989 a.u.) and 0.1453-

TABLE-3

TOTAL ELECTRON DENSITY $\rho$ (r), (a.u.) OF Pt-C AND Pt-N BONDS AT THE CALCULATED AND EXPERIMENTAL STRUCTURES USING DIFFERENT METHODS

\begin{tabular}{|c|c|c|c|c|c|c|c|c|c|c|c|c|c|c|}
\hline & \multicolumn{2}{|c|}{ B3LYP } & \multicolumn{2}{|c|}{ B3PW91 } & \multicolumn{2}{|c|}{ CAM-B3LYP } & \multicolumn{2}{|c|}{ M06 } & \multicolumn{2}{|c|}{ M06HF } & \multicolumn{2}{|c|}{ PBE } & \multicolumn{2}{|c|}{ MP2 } \\
\hline & Calc. & Exp. & Calc. & Exp. & Calc. & Exp. & Calc. & Exp. & Calc. & Exp. & Calc. & Exp. & Calc. & Exp. \\
\hline \multicolumn{15}{|c|}{ Complex 1} \\
\hline Pt-N25 & 0.0744 & 0.0929 & 0.0808 & 0.0934 & 0.0794 & 0.0921 & 0.0718 & 0.0920 & 0.0824 & 0.0918 & 0.0800 & 0.0945 & 0.0808 & 0.0894 \\
\hline Pt-N26 & 0.0782 & 0.0942 & 0.0843 & 0.0948 & 0.0824 & 0.0934 & 0.0756 & 0.0933 & 0.0839 & 0.0931 & 0.0830 & 0.0959 & 0.0822 & 0.0907 \\
\hline $\mathrm{Pt}-\mathrm{C} 1$ & 0.1477 & 0.1502 & 0.1522 & 0.1506 & 0.1510 & 0.1505 & 0.1462 & 0.1493 & 0.1657 & 0.1501 & 0.1488 & 0.1492 & 0.1678 & 0.1538 \\
\hline Pt-C6 & 0.1500 & 0.1543 & 0.1543 & 0.1547 & 0.1540 & 0.1547 & 0.1495 & 0.1534 & 0.1664 & 0.1541 & 0.1502 & 0.1533 & 0.1681 & 0.1580 \\
\hline \multicolumn{15}{|c|}{ Complex 2} \\
\hline Pt-N32 & 0.0731 & 0.0885 & 0.0795 & 0.0890 & 0.0780 & 0.0877 & 0.0696 & 0.0876 & 0.0795 & 0.0872 & 0.0785 & 0.0901 & 0.0803 & 0.0849 \\
\hline Pt-N33 & 0.0782 & 0.0972 & 0.0842 & 0.0977 & 0.0825 & 0.0963 & 0.0765 & 0.0962 & 0.0853 & 0.0961 & 0.0831 & 0.0989 & 0.0821 & 0.0936 \\
\hline $\mathrm{Pt}-\mathrm{C} 1$ & 0.1476 & 0.1556 & 0.1521 & 0.1560 & 0.1509 & 0.1559 & 0.1456 & 0.1546 & 0.1643 & 0.1555 & 0.1487 & 0.1546 & 0.1676 & 0.1594 \\
\hline Pt-C6 & 0.1501 & 0.1560 & 0.1545 & 0.1563 & 0.1543 & 0.1565 & 0.1505 & 0.1552 & 0.1676 & 0.1559 & 0.1506 & 0.1548 & 0.1681 & 0.1602 \\
\hline \multicolumn{15}{|c|}{ Complex 3} \\
\hline Pt-N27 & 0.0778 & 0.0929 & 0.0842 & 0.0935 & 0.0824 & 0.0921 & 0.0762 & 0.0921 & 0.0836 & 0.0918 & 0.0826 & 0.0945 & 0.0851 & 0.0894 \\
\hline Pt-N28 & 0.0778 & 0.0939 & 0.0842 & 0.0944 & 0.0824 & 0.0931 & 0.0762 & 0.0930 & 0.0836 & 0.0929 & 0.0826 & 0.0954 & 0.0851 & 0.0905 \\
\hline $\mathrm{Pt}-\mathrm{C} 1$ & 0.1487 & 0.1531 & 0.1532 & 0.1534 & 0.1521 & 0.1535 & 0.1475 & 0.1522 & 0.1660 & 0.1530 & 0.1498 & 0.1520 & 0.1679 & 0.1568 \\
\hline Pt-C10 & 0.1487 & 0.1512 & 0.1532 & 0.1515 & 0.1521 & 0.1515 & 0.1475 & 0.1502 & 0.1660 & 0.1511 & 0.1498 & 0.1502 & 0.1679 & 0.1548 \\
\hline \multicolumn{15}{|c|}{ Complex 4} \\
\hline Pt-N33 & 0.0677 & 0.0870 & 0.0744 & 0.0875 & 0.0706 & 0.0862 & 0.0653 & 0.0862 & 0.0787 & 0.0859 & 0.0728 & 0.0886 & 0.0779 & 0.0835 \\
\hline Pt-N34 & 0.0779 & 0.0958 & 0.0841 & 0.0963 & 0.0806 & 0.0949 & 0.0758 & 0.0948 & 0.0854 & 0.0946 & 0.0828 & 0.0975 & 0.0829 & 0.0921 \\
\hline Pt-C1 & 0.1481 & 0.1523 & 0.1525 & 0.1527 & 0.1506 & 0.1526 & 0.1458 & 0.1513 & 0.1645 & 0.1522 & 0.1494 & 0.1515 & 0.1673 & 0.1558 \\
\hline Pt-C6 & 0.1515 & 0.1562 & 0.1557 & 0.1565 & 0.1537 & 0.1567 & 0.1513 & 0.1554 & 0.1678 & 0.1562 & 0.1518 & 0.1550 & 0.1692 & 0.1606 \\
\hline \multicolumn{15}{|c|}{ Complex 5} \\
\hline Pt-N27 & 0.0687 & 0.0855 & 0.0758 & 0.0861 & 0.0742 & 0.0847 & 0.0664 & 0.0848 & 0.0790 & 0.0843 & 0.0753 & 0.0950 & 0.0761 & 0.0820 \\
\hline Pt-N28 & 0.0762 & 0.0933 & 0.0830 & 0.0939 & 0.0810 & 0.0925 & 0.0748 & 0.0924 & 0.0846 & 0.0921 & 0.0816 & 0.0872 & 0.0825 & 0.0896 \\
\hline $\mathrm{Pt}-\mathrm{C} 1$ & 0.1477 & 0.1556 & 0.1524 & 0.1559 & 0.1507 & 0.1558 & 0.1453 & 0.1545 & 0.1636 & 0.1554 & 0.1490 & 0.1546 & 0.1671 & 0.1590 \\
\hline Pt-C6 & 0.1515 & 0.1585 & 0.1554 & 0.1588 & 0.1555 & 0.1590 & 0.1510 & 0.1576 & 0.1679 & 0.1585 & 0.1511 & 0.1572 & 0.1702 & 0.1627 \\
\hline \multicolumn{15}{|c|}{ Complex 6} \\
\hline Pt-N34 & 0.0680 & 0.0851 & 0.0743 & 0.0856 & 0.0711 & 0.0843 & 0.0656 & 0.0843 & 0.0783 & 0.0838 & 0.0733 & 0.0867 & 0.0754 & 0.0815 \\
\hline Pt-N35 & 0.0774 & 0.0948 & 0.0838 & 0.0953 & 0.0802 & 0.0940 & 0.0756 & 0.0938 & 0.0862 & 0.0936 & 0.0824 & 0.0965 & 0.0829 & 0.0911 \\
\hline $\mathrm{Pt}-\mathrm{C} 1$ & 0.1483 & 0.1565 & 0.1530 & 0.1568 & 0.1508 & 0.1567 & 0.1457 & 0.1554 & 0.1639 & 0.1563 & 0.1497 & 0.1556 & 0.1681 & 0.1600 \\
\hline Pt-C6 & 0.1514 & 0.1588 & 0.1556 & 0.1591 & 0.1533 & 0.1593 & 0.1511 & 0.1579 & 0.1680 & 0.1588 & 0.1514 & 0.1575 & 0.1703 & 0.1632 \\
\hline
\end{tabular}




\begin{tabular}{|c|c|c|c|c|c|c|c|c|c|c|c|c|c|c|}
\hline \multicolumn{15}{|c|}{$\begin{array}{l}\text { TABLE-4 } \\
\text { TOTAL ENERGY DENSITY H(r) OF Pt-C AND Pt-N BONDS AT THE CALCULATED } \\
\text { AND EXPERIMENTAL STRUCTURES USING DIFFERENT METHODS }\end{array}$} \\
\hline & \multicolumn{2}{|c|}{ B3LYP } & \multicolumn{2}{|c|}{ B3PW91 } & \multicolumn{2}{|c|}{ CAM-B3LYP } & \multicolumn{2}{|c|}{ M06 } & \multicolumn{2}{|c|}{ M06HF } & \multicolumn{2}{|c|}{ PBE } & \multicolumn{2}{|c|}{ MP2 } \\
\hline & Calc. & Exp. & Calc. & Exp. & Calc. & Exp. & Calc. & Exp. & Calc. & Exp. & Calc. & Exp. & Calc. & Exp. \\
\hline \multicolumn{15}{|c|}{ Complex 1} \\
\hline Pt-N25 & -0.0138 & -0.0208 & 0.0164 & -0.0214 & -0.0154 & -0.0203 & -0.0129 & -0.0204 & -0.0173 & -0.0209 & -0.0165 & -0.0224 & -0.0143 & -0.0175 \\
\hline Pt-N26 & -0.0153 & -0.0217 & -0.0179 & -0.0223 & -0.0168 & -0.0212 & -0.0143 & -0.0211 & -0.0182 & -0.0220 & -0.0178 & -0.0233 & 0.0153 & -0.0185 \\
\hline Pt-C1 & -0.0653 & -0.0678 & -0.0695 & -0.0687 & -0.0679 & -0.0680 & -0.0638 & -0.0667 & -0.0822 & -0.0692 & -0.0664 & -0.0674 & -0.0844 & -0.0723 \\
\hline Pt-C6 & -0.0678 & -0.0711 & -0.0721 & -0.0721 & -0.0710 & -0.0714 & -0.0671 & -0.0700 & -0.0835 & -0.0724 & -0.0684 & -0.0706 & -0.0852 & -0.0757 \\
\hline \multicolumn{15}{|c|}{ Complex 2} \\
\hline Pt-N32 & 0134 & 0190 & 0.0160 & -0.0196 & -0.0150 & 0.0185 & -0.0122 & -0.0185 & -0.0161 & -0.0189 & -0.0160 & -0.0206 & -0.0141 & -0.0157 \\
\hline Pt-N33 & -0.0153 & -0.0229 & -0.0179 & -0.0235 & -0.0168 & -0.0223 & -0.0147 & -0.0223 & -0.0187 & -0.0231 & -0.0179 & -0.0246 & -0.0152 & -0.0196 \\
\hline Pt-C1 & -0.0652 & -0.0720 & -0.0695 & -0.0731 & -0.0678 & -0.0723 & -0.0633 & -0.0708 & -0.0811 & -0.0735 & -0.0663 & -0.0716 & -0.0842 & -0.0771 \\
\hline Pt-C6 & -0.0680 & 0.0730 & -0.0723 & -0.0740 & -0.0713 & -0.0734 & -0.0681 & -0.0719 & -0.0848 & -0.0744 & -0.0687 & -0.0724 & -0.0852 & -0.0782 \\
\hline \multicolumn{15}{|c|}{ Complex 3} \\
\hline Pt-N27 & 0151 & 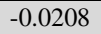 & .0178 & & -0.0167 & -0.0203 & -0.0144 & & -0.0 & $-0 . \mathrm{C}$ & & & & \\
\hline Pt-N28 & 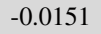 & & -0.0178 & & -0.01 & -0.0209 & & & -0.0 & -0.0 & -0.0 & & & \\
\hline Pt-C1 & -0.0662 & 0 & -0.0705 & -0. & -0.0689 & -0.0703 & -0 & & -0.0826 & -0.0714 & -0.0674 & $-0 .($ & -0. & -0. \\
\hline $\mathrm{Pt}-\mathrm{C} 10$ & -0.0662 & -0.0684 & -0.0705 & -0.0694 & -0.0689 & -0.0687 & -0.0650 & -0.0672 & -0.0826 & -0.0698 & -0.0674 & -0.0680 & -0.0847 & -0.0730 \\
\hline \multicolumn{15}{|c|}{ Complex 4} \\
\hline Pt-N33 & -0.0114 & -0.0183 & 0.0140 & -0.0189 & -0.0122 & -0.0178 & -0.0107 & -0.0180 & -0.0156 & -0.0184 & -0.0138 & -0.0199 & -0.0129 & -0.0151 \\
\hline Pt-N34 & -0.0151 & -0.0222 & -0.0178 & -0.0229 & -0.0160 & -0.0217 & -0.0143 & -0.0217 & -0.0187 & -0.0224 & -0.0177 & -0.0239 & -0.0155 & -0.0189 \\
\hline $\mathrm{Pt}-\mathrm{C} 1$ & -0.0654 & -0.0689 & -0.0696 & -0.0699 & -0.0671 & -0.0691 & -0.0633 & -0.0677 & -0.0811 & -0.0703 & -0.0666 & -0.0686 & -0.0837 & -0.0736 \\
\hline Pt-C6 & -0.0693 & -0.0732 & -0.0735 & -0.0742 & -0.0710 & -0.0736 & -0.0688 & -0.0721 & -0.0850 & -0.0748 & -0.0699 & -0.0726 & -0.0864 & -0.0786 \\
\hline \multicolumn{15}{|c|}{ Complex 5} \\
\hline Pt-N27 & 0118 & 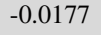 & 0144 & 82 & .0135 & -0.0172 & -0.0111 & 73 & -0.0 & -0.0176 & -0.0147 & $-0 .($ & -0.0125 & \\
\hline Pt-N28 & -0.0145 & -0.0210 & -0.0174 & -0.0216 & -0.0162 & -0.0205 & -0.0140 & -0.0205 & -0.0184 & -0.0212 & -0.0172 & -0.0192 & -0.0153 & -0.0177 \\
\hline Pt-C1 & -0.0652 & -0.0715 & -0.0696 & -0.0725 & -0.0674 & -0.0717 & -0.0630 & -0.0702 & -0.0797 & -0.0728 & -0.0664 & -0.0712 & -0.0836 & -0.0762 \\
\hline Pt-C6 & -0.0692 & -0.0750 & -0.0733 & -0.0760 & -0.0724 & -0.0754 & -0.0687 & -0.0738 & -0.0852 & -0.0765 & -0.0693 & -0.0743 & -0.0875 & -0.0803 \\
\hline \multicolumn{15}{|c|}{ Complex 6} \\
\hline Pt-N34 & -0.0116 & -0.0175 & -0.0139 & -0.0180 & -0.0124 & -0.0170 & -0.0108 & -0.0172 & -0.0155 & -0.0175 & -0.0140 & -0.0190 & -0.0122 & -0.0144 \\
\hline Pt-N35 & -0.0150 & -0.0217 & -0.0177 & -0.0223 & -0.0159 & -0.0212 & -0.0143 & -0.0212 & -0.0191 & -0.0218 & -0.0175 & -0.0234 & -0.0154 & -0.0183 \\
\hline $\mathrm{Pt}-\mathrm{C} 1$ & -0.0655 & -0.0723 & -0.0700 & -0.0734 & -0.0673 & -0.0725 & -0.0632 & -0.0710 & -0.0799 & -0.0737 & -0.0669 & -0.0720 & -0.0844 & -0.0772 \\
\hline Pt-C6 & -0.0692 & -0.0754 & -0.0735 & -0.0765 & -0.0707 & -0.0759 & -0.0688 & -0.0743 & -0.0854 & -0.0771 & -0.0696 & -0.0747 & -0.0877 & -0.0811 \\
\hline
\end{tabular}

TABLE-5

THE RATIO IV(r)|/G(r) OF Pt-C AND Pt-N BONDS AT THE CALCULATED AND EXPERIMENTAL STRUCTURES USING DIFFERENT METHODS

\begin{tabular}{|c|c|c|c|c|c|c|c|c|c|c|c|c|c|c|}
\hline & \multicolumn{2}{|c|}{ B3LYP } & \multicolumn{2}{|c|}{ B3PW91 } & \multicolumn{2}{|c|}{ CAM-B3LYP } & \multicolumn{2}{|c|}{ M06 } & \multicolumn{2}{|c|}{ M06HF } & \multicolumn{2}{|c|}{ PBE } & \multicolumn{2}{|c|}{ MP2 } \\
\hline & Calc. & Exp. & Calc. & Exp. & Calc. & Exp. & Calc. & Exp. & Calc. & Exp. & Calc. & Exp. & Calc. & Exp. \\
\hline \multicolumn{15}{|c|}{ Complex 1} \\
\hline Pt-N25 & 1512 & 1685 & 1630 & 1.1735 & 1.1527 & 1.1630 & 1.1458 & 1.1656 & 1.1578 & .1655 & 1.1728 & .1851 & 1.1257 & .1335 \\
\hline Pt-N26 & 1.1608 & 1765 & 1724 & 1.1814 & 1.1616 & 1.1709 & 1.1558 & 1724 & 1.1658 & 1.1750 & 1.1819 & 1.1934 & 1.1338 & 1.1425 \\
\hline Pt-C1 & 1.5531 & 5696 & 5667 & 1.5821 & 1.5496 & 1.5648 & 1.5465 & & 1.6128 & & 1.5601 & 1.5814 & 1.5414 & 1.5313 \\
\hline Pt-C6 & 1.5829 & 5747 & 1.5984 & 1.5874 & 1.5790 & 1.5694 & 1.5786 & 1.5670 & 1.6372 & 1.6060 & 1.5906 & 1.5869 & 1.5587 & 1.5332 \\
\hline \multicolumn{15}{|c|}{ Complex 2} \\
\hline Pt-1 & 499 & 8 & 1615 & 1.1678 & 1.15 & 1.1573 & 1.1434 & 1.1 & 1.1538 & 88 & 1.1715 & 8 & 1.1242 & 1.1270 \\
\hline Pt-N33 & 1.1612 & 1785 & 1726 & 1.1834 & 1.16 & 1.1727 & 1.1 & & 1.1671 & 1.1760 & 1.1823 & 54 & .1336 & 1.1438 \\
\hline $\mathrm{Pt}-\mathrm{C} 1$ & 1.5520 & 5725 & 1.5656 & 1.5851 & 1.5482 & 1.5676 & 1.5442 & 1.5639 & 1.6108 & 1.6076 & 1.5587 & 1.5844 & 1.5397 & 1.5409 \\
\hline & 1.5860 & .5948 & 1.6019 & 1.6077 & 1.5831 & 1.5915 & 1.5851 & 1.5888 & 1.6469 & 1.6327 & 1.5938 & 1.6043 & 1.5622 & 1.5579 \\
\hline \multicolumn{15}{|c|}{ Complex 3} \\
\hline $\bar{P}$ & 1586 & 1697 & 1708 & 1.1747 & 1.1598 & 1.1643 & 1.15 & 1.1667 & 1.1629 & 1.1664 & 1.1791 & 1.1 & 1.1346 & 1.1344 \\
\hline Pt-N28 & 1.1586 & 1738 & 1.1708 & 1.1789 & 1.1598 & 1.1688 & 1.1 & 03 & 1.1629 & 1.1729 & 1.1791 & 1.1898 & .1346 & 1.1406 \\
\hline Pt-C1 & 1.5605 & .5697 & 1.5744 & 1.5820 & 1.5564 & 1.5657 & 1.5557 & 1.5624 & 1.6199 & 1.6044 & 1.5684 & 1.5805 & 1.5444 & 1.5326 \\
\hline Pt-C10 & 1.5605 & .5689 & 1.5744 & 1.5812 & 1.5564 & 1.5643 & 1.5557 & 1.5603 & 1.6199 & 1.6033 & 1.5684 & 1.5803 & 1.5444 & 1.5329 \\
\hline \multicolumn{15}{|c|}{ Complex 4} \\
\hline$\sqrt{33}$ & 405 & 1610 & 1.1525 & .1658 & 1.1392 & 1.1557 & 1.1358 & 1.1581 & 1.1494 & 1.1584 & 1.1615 & 1.1773 & 1.1172 & 1.1256 \\
\hline Pt-N34 & 1.1601 & 1.1773 & 1.1718 & 1.1822 & 1.1592 & 1.1714 & 1.1550 & 1.1734 & 1.1671 & 1.1739 & 1.1811 & 1.1944 & 1.1343 & 1.1409 \\
\hline Pt-C1 & 1.5463 & 1.5539 & 1.5592 & 1.5663 & 1.5335 & 1.5485 & 1.5401 & 1.5452 & 1.6053 & 1.5861 & 1.5529 & 1.5670 & 1.5305 & 1.5211 \\
\hline Pt-C6 & 1.5952 & 1.5971 & 1.6103 & 1.6102 & 1.5922 & 1.5946 & 1.5926 & 1.5912 & 1.6491 & 1.6378 & 1.6014 & 1.6063 & 1.5713 & 1.5628 \\
\hline \multicolumn{15}{|c|}{ Complex 5} \\
\hline$\sqrt{27}$ & 420 & 1580 & 1.1545 & 1.1629 & 1.1 & 1528 & 1.1381 & 55 & 1.1513 & 47 & 1.1646 & .1898 & .1169 & 1.1237 \\
\hline Pt-N28 & 1.1580 & 1.1725 & 1.1702 & 1.1774 & 1.1597 & 1.1668 & 1.1538 & 1.1688 & 1.1676 & 1.1690 & 1.1794 & 1.1746 & 1.1338 & 1.1364 \\
\hline & 1.5477 & 1.5595 & 1.5621 & 1.5720 & 1.5411 & 1.5531 & 1.5396 & 1.5502 & 1.5898 & 1.5887 & 1.5575 & 1.5734 & 1.5309 & 1.5232 \\
\hline $\mathrm{Pt}-\mathrm{C} 6$ & 1.5951 & 1.5963 & 1.6100 & 1.6093 & 1.5928 & 1.5933 & 1.5925 & 1.5904 & 1.6526 & 1.6340 & 1.5995 & 1.6050 & 1.5756 & 1.5585 \\
\hline
\end{tabular}




\begin{tabular}{|c|c|c|c|c|c|c|c|c|c|c|c|c|c|c|}
\hline \multicolumn{15}{|c|}{ Complex 6} \\
\hline Pt-N34 & 1.1415 & 1.1576 & 1.1531 & 1.1624 & 1.1407 & 1.1524 & 1.1373 & 1.1549 & 1.1505 & 1.1542 & 1.1627 & 1.1741 & 1.1162 & 1.1232 \\
\hline Pt-N35 & 1.1597 & 1.1741 & 1.1713 & 1.1790 & 1.1590 & 1.1684 & 1.1551 & 1.1704 & 1.1692 & 1.1708 & 1.1806 & 1.1912 & 1.1340 & 1.1381 \\
\hline $\mathrm{Pt}-\mathrm{C} 1$ & 1.5435 & 1.5626 & 1.5577 & 1.5753 & 1.5320 & 1.5563 & 1.5354 & 1.5535 & 1.5837 & 1.5932 & 1.5532 & 1.5765 & 1.5294 & 1.5274 \\
\hline Pt-C6 & 1.5976 & 1.6044 & 1.6134 & 1.6175 & 1.5939 & 1.6020 & 1.5953 & 1.5984 & 1.6578 & 1.6459 & 1.6027 & 1.6125 & 1.5784 & 1.5693 \\
\hline
\end{tabular}

\begin{tabular}{|c|c|c|c|c|c|c|c|c|c|c|c|c|c|c|}
\hline \multicolumn{15}{|c|}{$\begin{array}{l}\text { TABLE-6 } \\
\text { LAPLACIAN OF THE ELECTRON DENSITY }\left(\nabla^{2} \rho(r)\right) \text { OF Pt-C AND Pt-N BONDS AT THE } \\
\text { CALCULATED AND EXPERIMENTAL STRUCTURES USING DIFFERENT METHODS }\end{array}$} \\
\hline & \multicolumn{2}{|c|}{ B3LYP } & \multicolumn{2}{|c|}{ B3PW91 } & \multicolumn{2}{|c|}{ CAM-B3LYP } & \multicolumn{2}{|c|}{ M06 } & \multicolumn{2}{|c|}{ M06HF } & \multicolumn{2}{|c|}{ PBE } & \multicolumn{2}{|c|}{ MP2 } \\
\hline & Calc. & Exp. & Calc. & Exp. & Calc. & Exp. & Calc. & Exp. & Calc. & Exp. & Calc. & Exp. & Calc. & Exp. \\
\hline \multicolumn{15}{|c|}{ Complex 1} \\
\hline Pt-N25 & 0.3097 & r & 3374 & 0.70 & 0.3420 & 0.4161 & 0.3014 & 0 . & 0.5002 & & 0.5100 & 0 & 3986 & 4543 \\
\hline Pt-N26 & 0.3192 & 4048 & 3444 & 0.4025 & 0.3484 & 0.4104 & 0.3107 & & 0.3655 & & 3206 & 0.3893 & 3950 & 0.4462 \\
\hline Pt-C1 & 0.2109 & .2048 & 0.2127 & 0.1974 & 0.2226 & 0.2097 & 0.2119 & 0.2079 & 0.2078 & 0.1817 & 0.2086 & 0.1940 & 2861 & 0.2550 \\
\hline Pt-C6 & 0.1941 & 2104 & 0.1935 & .2025 & 0.2065 & 0.2159 & 0.1956 & 0.2137 & 0.1902 & 0.1882 & 0.1897 & 0.1989 & 0.2690 & 0.2650 \\
\hline \multicolumn{15}{|c|}{ Complex 2} \\
\hline Pt-N32 & 035 & 3 & 315 & 0.38 & 0.3369 & 0.3956 & 0.2911 & 0.3 & 3550 & 0.4 & 0.3100 & & 80 & 11 \\
\hline Pt-N33 & 0.3186 & 4214 & 0.3432 & 0.4188 & 0.3482 & 0.4278 & 0.3152 & 0.4220 & 0.3726 & 0.3058 & 0.3205 & 0.4046 & 3944 & 0.4676 \\
\hline Pt-C1 & 0.2116 & 2152 & 0.2134 & 0.2073 & 0.2234 & 0.2204 & 0.2121 & 0.2191 & 0.2066 & 0.1899 & 0.2094 & 0.2037 & 0.2873 & 0.2619 \\
\hline Pt-C6 & 0.1921 & 1988 & 0.1913 & 0.1910 & 0.2039 & 0.2027 & 0.1932 & 0.2010 & 0.1851 & 0.1728 & 0.1880 & 0.1 & 0.2655 & 0.2478 \\
\hline \multicolumn{15}{|c|}{ Complex 3} \\
\hline $10-102$ & & & & & & & 66 & & 3676 & & 3222 & & & \\
\hline Pt-N28 & 0.3195 & 0.4073 & 3464 & 0.4049 & 0.3507 & 0.4126 & 0.3168 & 0.4081 & 0.3676 & 0.4168 & 0.3222 & 0.3923 & 4155 & 0.4490 \\
\hline Pt-C1 & 0.2076 & 0.2114 & 0.2091 & 0.2038 & 0.2196 & 0.2159 & 0.2079 & 0.2142 & 0.2027 & 0.1870 & 0.2046 & 0.2009 & 0.2834 & 0.2622 \\
\hline Pt-C10 & 0.2076 & .2073 & 0.2091 & 0.1999 & 0.2196 & 0.2121 & 0.2079 & 0.2110 & 0.2027 & 0.1837 & 0.2045 & 0.1966 & 0.2834 & 0.2560 \\
\hline \multicolumn{15}{|c|}{ Complex 4} \\
\hline Pt-N33 & & & & & & & 0.2726 & & & & 0.2866 & & & \\
\hline Pt-N34 & 0.3174 & 0.4129 & 0.3433 & 0.4104 & 0.3374 & 0.4194 & 0.3124 & 0.4137 & 0.3725 & 0.4257 & 0.3198 & 0.3962 & 0.3986 & 0.4607 \\
\hline $\mathrm{Pt}-\mathrm{C} 1$ & 0.2172 & 0.2219 & 0.2195 & 0.2141 & 0.2349 & 0.2275 & 0.2158 & 0.2258 & 0.2115 & 0.1985 & 0.2155 & 0.2094 & 0.2963 & 0.2704 \\
\hline Pt-C6 & 0.1884 & 0.1975 & 0.1877 & 0.1896 & 0.1956 & 0.2008 & 0.1893 & 0.1995 & 0.1838 & 0.1698 & 0.1852 & 0.1885 & 0.2595 & 0.2443 \\
\hline \multicolumn{15}{|c|}{ Complex 5} \\
\hline Pt-N27 & 0.2843 & 0.3762 & 0.3161 & 0.3742 & 0.3195 & 0.3808 & 0.2767 & 0.3764 & 0.3532 & 0.3856 & 0.2979 & 0.3875 & .3762 & 0.4124 \\
\hline Pt-N28 & 0.3100 & 0.4037 & 0.3391 & 0.4013 & 0.3409 & 0.4098 & 0.3081 & 0.4042 & 0.3660 & 0.4161 & 0.3154 & 0.3627 & 0.3967 & 0.4491 \\
\hline $\mathrm{Pt}-\mathrm{C} 1$ & 0.2153 & 0.2251 & 0.2167 & 0.2170 & 0.2287 & 0.2317 & 0.2150 & & 0.2219 & 0.2034 & 0.2110 & 0.2118 & 0.2955 & 0.2776 \\
\hline Pt-C6 & 0.1884 & 0.2029 & 0.1873 & 0.1949 & 0.1990 & 0.2067 & 0.1890 & 0.2050 & 0.1813 & 0.1766 & 0.1851 & 0.1941 & 0.2580 & 0.2540 \\
\hline \multicolumn{15}{|c|}{ Complex 6} \\
\hline Pt-N34 & 0.2807 & 0.3743 & 3086 & 0.3723 & 0.3028 & 0.3789 & 0.2722 & 0.3747 & 0.3495 & 0.3836 & 0.2881 & 0.3609 & 3718 & 0.4103 \\
\hline Pt-N35 & 0.3150 & 0.4118 & 0.3424 & 0.4093 & 0.3354 & 0.4179 & 0.3111 & 0.4123 & 0.3741 & 0.4241 & 0.3183 & 0.3953 & 0.3988 & 0.4580 \\
\hline $\mathrm{Pt}-\mathrm{C} 1$ & 0.2201 & 0.2250 & 0.2220 & 0.2167 & 0.2367 & 0.2314 & 0.2192 & 0.2292 & 0.2281 & 0.2021 & 0.2160 & 0.2116 & 0.3002 & 0.2765 \\
\hline Pt-C6 & 0.1864 & 0.1975 & 0.1853 & 0.1894 & 0.1934 & 0.2007 & 0.1871 & 0.1996 & 0.1776 & 0.1690 & 0.1835 & 0.1891 & 0.2556 & 0.2453 \\
\hline
\end{tabular}

0.1680 a.u. (exp. 0.1493-0.1632a.u.), respectively [31]. The studied Pt complexes (except 3 ) have two types of $\mathrm{Pt}-\mathrm{N}$ bonds i.e., $\mathrm{Pt}-\mathrm{N}$ (hydrazone) and $\mathrm{Pt}-\mathrm{N}$ (pyridine). Based on the $\rho(\mathrm{r})$ values, the interaction of $\mathrm{Pt}^{2+}$ with the $\mathrm{N}$-atom of pyridine is stronger than that with the $\mathrm{N}$-atom of hydrazone for all the studied compounds. The $\rho(r)$ values for the Pt-C BCPs are generally higher than 0.1 a.u, with $\mathrm{Pt}-\mathrm{C}$ bonds trans to $\mathrm{Pt}-\mathrm{N}$ (pyridine) bonds considered weaker than $\mathrm{Pt}-\mathrm{C}$ bonds trans to $\mathrm{Pt}-\mathrm{N}$ (hydrazone) bonds.

To predict the effect of substituent at the hydrazone moiety on the Pt-N bond characteristics, we selected complex $\mathbf{3}$ for comparison purposes. Interestingly, the average electron density, $\rho(\mathrm{r})$, of the two Pt-N bonds is the highest in 3. The other compounds have one pyridine ring attached to the hydrazone moiety with different substituents at the hydrazone moiety. Complexes $\mathbf{1}$ and $\mathbf{2}$ have methyl and phenyl substituents, respectively, at the carbon $(\mathrm{C}=\mathrm{N})$ of the hydrazone moiety. In this case, lower $\rho(r)$ values were observed for $\mathbf{2}$ than for $\mathbf{1}$. In contrast, complexes 4-6 have N-substituents at the hydrazone moiety. The $\rho(\mathrm{r})$ values of $\mathrm{N}$-substituted hydrazones are lower than those for C-substituted hydrazones. As a result, the interaction between $\mathrm{Pt}$ and the $\mathrm{N}$-atom of hydrazone is weakest for compounds 4-6. The degree by which the $\rho(r)$ values are decreased depends on the nature of substituent at the amino group. The maximum decrease is observed for the F-benzoyl derivative (6) and hence, the weakest $\mathrm{Pt}-\mathrm{N}$ interaction is predicted. In agreement with the high electron-withdrawing character of the F-benzoyl group, which decreases the ability of the $\mathrm{N}$-atom lone pair to interact with the metal ion, a weak $\mathrm{Pt}-\mathrm{N}$ interaction is predicted for $\mathbf{6}$. The opposite is true for $\mathbf{1}$, in which the methyl group acts as an electron-donating group via the inductive effect.

In contrast to Pt-N (hydrazone) bonds, $\mathrm{Pt}-\mathrm{N}$ (pyridine) bonds are not greatly affected by varying the substituent. Among the examined complexes, complex 2 shows the highest electron density at the Pt-N (pyridine) BCP. Replacement of the phenyl group in $\mathbf{2}$ by a methyl group (1) slightly lowers the $\rho(\mathrm{r})$ values. In comparison, phenyl (4), acetyl (5) and F-benzoyl (6) substi- 
tuents significantly lower the $\rho(\mathrm{r})$ values of the $\mathrm{Pt}-\mathrm{N}$ bonds. Similar to the behaviour observed for Pt-N (hydrazone) bonds, the Pt-N (pyridine) interactions of the latter compounds are expected to be the weakest.

For hydrogen bonding systems, the hydrogen bond energy $\left(E_{\text {int }}\right)$ can be estimated from the potential energy density [V(r)] at the corresponding BCP using the relation $\mathrm{E}_{\text {int }}=\mathrm{V}(\mathrm{r}) / 2$ [32]. In these studies, the magnitude of the interaction energy was used as a measure of the hydrogen bond strength. By the same principle, this relation can be used to express the interaction energies at $\mathrm{Pt}-\mathrm{N}$ and $\mathrm{Pt}-\mathrm{C}, \mathrm{BCPs}$ to quantitatively assess $\mathrm{Pt}-\mathrm{N}$ and $\mathrm{Pt}-\mathrm{C}$ bond strength (Table-7). The $\mathrm{Pt}-\mathrm{N}$ (pyridine) bonds have higher interaction energies than the $\mathrm{Pt}-\mathrm{N}$ (hydrazone) bonds, whereas the two Pt-C bonds have similar $\mathrm{E}_{\text {int }}$ values. Fig. 2 shows the relation between the interaction energies at the $\mathrm{BCP}$ and the $\mathrm{Pt}-\mathrm{N}$ and $\mathrm{Pt}-\mathrm{C}$ bond distances. It is clear that the interaction energies are larger for shorter bonds.

\begin{tabular}{|c|c|c|c|c|c|c|c|c|c|c|c|c|c|c|}
\hline \multicolumn{15}{|c|}{$\begin{array}{l}\text { TABLE-7 } \\
\text {; (kcal/mol) OF Pt-C AND Pt-N BONDS AT THE } \\
\text { L STRUCTURES USING DIFFERENT METHODS }\end{array}$} \\
\hline & \multicolumn{2}{|c|}{ B3LYP } & \multicolumn{2}{|c|}{ B3PW91 } & \multicolumn{2}{|c|}{ CAM-B3LYP } & \multicolumn{2}{|c|}{ M06 } & \multicolumn{2}{|c|}{ M06HF } & \multicolumn{2}{|c|}{ PBE } & \multicolumn{2}{|c|}{ MP2 } \\
\hline & Calc. & Exp. & Calc. & Exp. & Calc. & Exp. & Calc. & Exp. & Calc. & Exp. & Calc. & Exp. & Calc. & Exp. \\
\hline \multicolumn{15}{|c|}{ Complex 1} \\
\hline Pt-N25 & 32.95 & 45.24 & 36.77 & 45.44 & 36.58 & 45.35 & 31.71 & 44.98 & 39.71 & 46.24 & 35.20 & 45.04 & 40.25 & 46.62 \\
\hline Pt-N26 & 34.63 & 45.36 & 38.26 & 45.56 & 37.86 & 45.47 & 33.36 & 45.09 & 40.06 & 46.27 & 36.34 & 45.18 & 40.56 & 46.63 \\
\hline $\mathrm{Pt}-\mathrm{C} 1$ & 57.49 & 58.59 & 60.32 & 58.61 & 60.07 & 59.15 & 56.66 & 58.13 & 67.88 & 57.65 & 58.03 & 57.49 & 75.43 & 65.34 \\
\hline Pt-C6 & 57.78 & 61.11 & 60.40 & 61.13 & 60.75 & 61.72 & 57.46 & 60.66 & 67.32 & 60.17 & 57.80 & 59.93 & 74.53 & 68.26 \\
\hline \multicolumn{15}{|c|}{ Complex 2} \\
\hline Pt-N32 & 32.19 & 42.52 & 36.02 & 42.73 & 35.83 & 42.61 & 30.48 & 42.28 & 37.97 & 43.37 & 34.38 & 42.37 & 40.07 & 43.66 \\
\hline Pt-N33 & 34.60 & 47.42 & 38.16 & 47.61 & 37.88 & 47.56 & 33.93 & 47.11 & 40.95 & 48.50 & 36.35 & 47.15 & 40.48 & 49.00 \\
\hline $\mathrm{Pt}-\mathrm{C} 1$ & 57.50 & 62.09 & 60.32 & 62.12 & 60.07 & 62.67 & 56.37 & 61.61 & 67.07 & 61.03 & 58.02 & 60.93 & 75.37 & 68.94 \\
\hline Pt-C6 & 57.72 & 61.39 & 60.37 & 61.41 & 60.72 & 61.95 & 57.88 & 60.91 & 67.71 & 60.25 & 57.87 & 60.28 & 74.31 & 68.50 \\
\hline \multicolumn{15}{|c|}{ Complex 3} \\
\hline Pt-N27 & 34.51 & 44.97 & 38.36 & 45.17 & 37.99 & 45.11 & 33.89 & 44.73 & 40.05 & 46.02 & 36.30 & 44.74 & 42.72 & 46.43 \\
\hline Pt-N28 & 34.52 & 45.39 & 38.37 & 45.60 & 37.97 & 45.51 & 33.91 & 45.16 & 40.06 & 46.36 & 36.30 & 45.18 & 42.73 & 46.75 \\
\hline $\mathrm{Pt}-\mathrm{C} 1$ & 57.82 & 60.49 & 60.67 & 60.52 & 60.45 & 61.06 & 57.11 & 59.99 & 67.75 & 59.50 & 58.31 & 59.36 & 75.35 & 67.44 \\
\hline Pt-C10 & 57.82 & 59.17 & 60.67 & 59.21 & 60.45 & 59.74 & 57.11 & 58.73 & 67.76 & 58.23 & 58.31 & 58.07 & 75.37 & 65.89 \\
\hline \multicolumn{15}{|c|}{ Complex 4} \\
\hline Pt-N33 & 29.14 & 41.48 & 33.09 & 41.68 & 31.32 & 41.57 & 28.10 & 41.30 & 37.55 & 42.29 & 31.14 & 41.36 & 38.69 & 42.53 \\
\hline Pt-N34 & 34.39 & 46.35 & 38.09 & 46.54 & 36.49 & 46.51 & 33.50 & 46.06 & 40.94 & 47.45 & 36.17 & 46.08 & 40.97 & 47.99 \\
\hline $\mathrm{Pt}-\mathrm{C} 1$ & 58.07 & 60.63 & 60.90 & 60.66 & 60.56 & 61.22 & 56.68 & 60.17 & 67.46 & 59.68 & 58.72 & 59.45 & 75.77 & 67.38 \\
\hline Pt-C6 & 58.24 & 61.41 & 60.84 & 61.43 & 59.90 & 61.96 & 58.05 & 60.90 & 67.76 & 60.25 & 58.36 & 60.31 & 74.59 & 68.50 \\
\hline \multicolumn{15}{|c|}{ Complex $\mathbf{5}$} \\
\hline Pt-N27 & 29.68 & 40.59 & 33.86 & 40.78 & 33.51 & 40.64 & 28.66 & 40.40 & 37.58 & 41.32 & 32.57 & 44.63 & 37.32 & 41.48 \\
\hline Pt-N28 & 33.44 & 44.87 & 37.52 & 45.06 & 36.91 & 45.01 & 32.95 & 44.59 & 40.27 & 45.91 & 35.56 & 40.49 & 40.73 & 46.35 \\
\hline $\mathrm{Pt}-\mathrm{C} 1$ & 57.77 & 62.52 & 60.64 & 62.54 & 60.23 & 63.15 & 56.38 & 62.04 & 67.45 & 61.63 & 58.24 & 61.29 & 75.63 & 69.57 \\
\hline Pt-C6 & 58.22 & 62.96 & 60.67 & 62.98 & 61.07 & 63.53 & 57.94 & 62.42 & 67.66 & 61.86 & 57.99 & 61.85 & 75.13 & 70.32 \\
\hline \multicolumn{15}{|c|}{ Complex 6} \\
\hline Pt-N34 & 29.27 & 40.34 & 32.96 & 40.53 & 31.54 & 40.40 & 28.15 & 40.16 & 37.13 & 41.06 & 31.38 & 40.25 & 36.84 & 41.22 \\
\hline Pt-N35 & 34.10 & 45.91 & 37.96 & 46.11 & 36.26 & 46.06 & 33.36 & 45.62 & 41.30 & 46.97 & 35.97 & 45.67 & 40.96 & 47.44 \\
\hline $\mathrm{Pt}-\mathrm{C} 1$ & 58.37 & 63.04 & 61.33 & 63.06 & 60.79 & 63.67 & 56.83 & 62.56 & 68.05 & 62.09 & 58.91 & 61.80 & 76.53 & 70.11 \\
\hline Pt-C6 & 58.05 & 62.82 & 60.65 & 62.84 & 59.55 & 63.37 & 57.84 & 62.30 & 67.50 & 61.62 & 58.05 & 61.72 & 75.06 & 70.10 \\
\hline
\end{tabular}
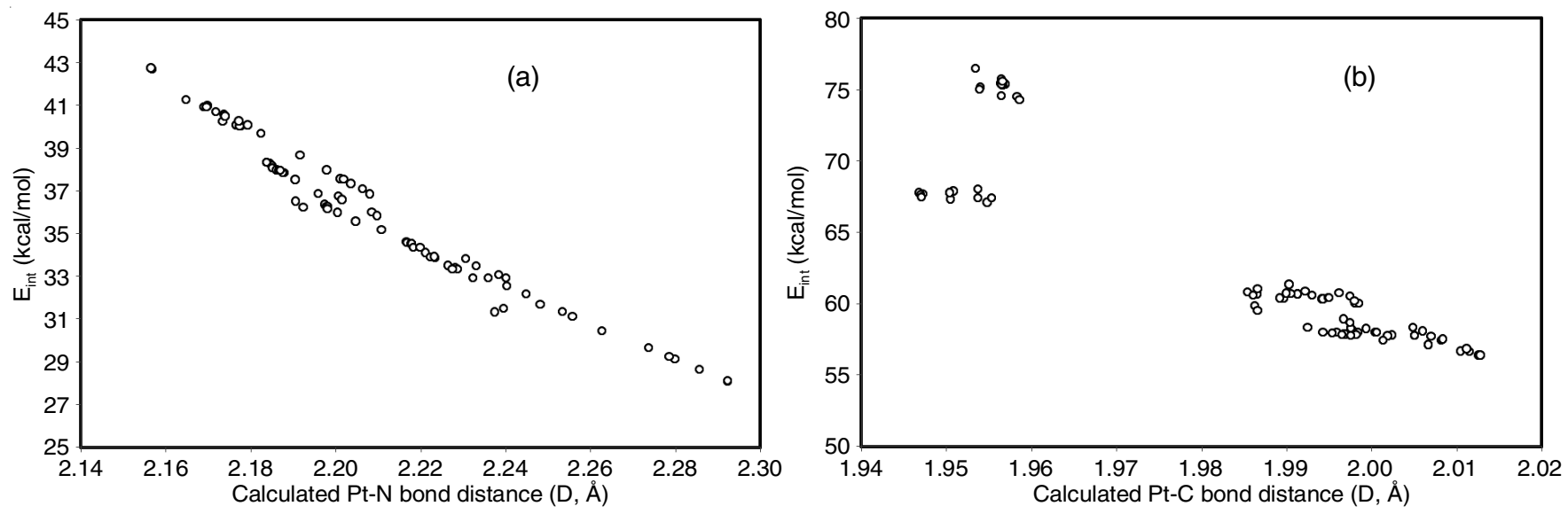

Fig. 2. Inverse relations between interaction energy and (a) $\mathrm{Pt}-\mathrm{N}$ and (b) $\mathrm{Pt}-\mathrm{C}$ bond distances 
The laplacian of electron density, $\nabla^{2} \rho(\mathrm{r})$, at the BCP is considered as another topological parameter that indicates bonding nature. Covalent interactions are characterized by a concentration of electron density between the interacting atoms and in this case, $\nabla^{2} \rho(r)$ is negative with a magnitude of the order of 1 a.u. In contrast, when the electron density is concentrated at each of the interacting nuclei, the laplacian is positive (about 0.1 a.u.), which is characteristic of closed-shell interactions [16]. However, these rules are violated in many cases, and positive values of the laplacian have been observed for covalent interactions. In agreement with this, the results shown in Table-6 indicate that $\nabla^{2} \rho(\mathrm{r})$ is positive for all $\mathrm{Pt}-\mathrm{N}$ and $\mathrm{Pt}-\mathrm{C}$ bonds.

The sum of the kinetic energy density $[\mathrm{G}(\mathrm{r})]$ and potential energy density $[\mathrm{V}(\mathrm{r})]$ gives the total energy density $[\mathrm{H}(\mathrm{r})]$, which is commonly used to characterize the nature of bonding [33]. $\mathrm{H}(\mathrm{r})$ is negative for covalent bonding and positive for closed-shell interactions. In addition, the ratio $\mid \mathrm{V}(\mathrm{r}) / / \mathrm{G}(\mathrm{r})$ is a significant parameter for characterizing the nature of bonding. Typically, the value of this ratio is more than 2 for covalent interactions [34]. In general, $\mathrm{IV}(\mathrm{r}) / / \mathrm{G}(\mathrm{r})>1$ indicates that covalent bonding is dominant, whereas $\mid \mathrm{V}(\mathrm{r}) / / \mathrm{G}(\mathrm{r})<1$ indicates that closed-shell interactions are predominant. All $\mathrm{Pt}-\mathrm{N}$ and $\mathrm{Pt}-\mathrm{C}$ bonds have negative $\mathrm{H}(\mathrm{r})$ values and $\mathrm{IV}(\mathrm{r}) / / \mathrm{G}(\mathrm{r})$ ratios of greater than 1 . Furthermore, more negative $\mathrm{H}(\mathrm{r})$ values and higher $\mathrm{IV}(\mathrm{r}) / / \mathrm{G}(\mathrm{r})$ ratios are obtained for $\mathrm{Pt}-\mathrm{N}$ (pyridine) bonds than for Pt-N (hydrazone) bonds. Similarly, Pt-C bonds trans to $\mathrm{Pt}-\mathrm{N}$ (hydrazone) are more like to be covalent than $\mathrm{Pt}-\mathrm{C}$ bonds trans to Pt-N (pyridine) bonds.

The ellipticity $(\varepsilon)$ at the $\mathrm{BCP}$ is a sensitive topological parameter used to evaluate the $\pi$-character of bonds [35]. This parameter indicates the degree of distortion in the electron density at the line perpendicular to the bond. Its value is 0.000 at the $\mathrm{C}-\mathrm{C} \mathrm{BCP}$ in ethane and increases with increasing $\pi$ character upto the double bond system [35]. In ethylene, the value of $\varepsilon$ is 0.332 . Table- 8 shows the ellipticity values of the $\mathrm{Pt}-\mathrm{C}$ and $\mathrm{Pt}-\mathrm{N}$ bonds obtained using different computational methods. The data clearly indicate the $\pi$-character of the Pt-N and $\mathrm{Pt}-\mathrm{C}$ bonds, with ellipticity values of upto 0.2 indicative of significant $\pi$-contributions in these bonds, although there are some discrepancies between the different methods.

NBO charges: NBO analysis is one of the most common and accurate methods for predicting atomic charges in molecular systems. NBO charges are less sensitive to basis set changes than other population analyses. Herein, we used the NBO method to compute the natural charges at the different atomic sites to describe the electron transfer processes between the Pt-ion and the ligand system. Moreover, the natures of the orbitals included in the Pt-N and Pt-C bonds were also investigated.

Table-9 shows the net natural charges at the acetyl groups, ligand moiety and $\mathrm{Pt}^{2+}$ ion of the studied complexes, both at the experimental and calculated geometries. It is clear that all

TABLE-8

THE ELLIPTICITY ( $(\varepsilon)$ OF Pt-C AND Pt-N BONDS AT THE CALCULATED AND EXPERIMENTAL STRUCTURES USING DIFFERENT METHODS

\begin{tabular}{|c|c|c|c|c|c|c|c|c|c|c|c|c|c|c|}
\hline & \multicolumn{2}{|c|}{ B3LYP } & \multicolumn{2}{|c|}{ B3PW91 } & \multicolumn{2}{|c|}{ CAM-B3LYP } & \multicolumn{2}{|c|}{ M06 } & \multicolumn{2}{|c|}{ M06HF } & \multicolumn{2}{|c|}{ PBE } & \multicolumn{2}{|c|}{ MP2 } \\
\hline & Calc. & Exp. & Calc. & Exp. & Calc. & Exp. & Calc. & Exp. & Calc. & Exp. & Calc. & Exp. & Calc. & Exp. \\
\hline \multicolumn{15}{|c|}{ Complex 1} \\
\hline Pt-N25 & 0.1436 & 0.0966 & 0.1307 & 0.0936 & 0.1295 & 0.0909 & 0.1237 & 0.0882 & 0.0921 & 0.0754 & 0.1325 & 0.0996 & 0.1530 & 0.1018 \\
\hline Pt-N26 & 0.0733 & 0.1014 & 0.0627 & 0.0977 & 0.0665 & 0.0985 & 0.0679 & 0.0908 & 0.0768 & 0.0902 & 0.0575 & 0.1002 & 0.1156 & 0.1247 \\
\hline $\mathrm{Pt}-\mathrm{C} 1$ & 0.0835 & 0.0963 & 0.0848 & 0.0962 & 0.0776 & 0.0942 & 0.0834 & 0.0922 & 0.0715 & 0.0869 & 0.0899 & 0.0988 & 0.0705 & 0.0999 \\
\hline Pt-C6 & 0.1050 & 0.0964 & 0.1061 & 0.0959 & 0.1026 & 0.0959 & 0.0981 & 0.0924 & 0.0834 & 0.0895 & 0.1092 & 0.0970 & 0.1098 & 0.1065 \\
\hline \multicolumn{15}{|c|}{ Complex 2} \\
\hline Pt-N32 & 0.1457 & 0.1586 & 0.1332 & 0.1539 & 0.1311 & 0.1540 & 0.1346 & 0.1439 & 0.1215 & 0.1333 & 0.1383 & 0.1579 & 0.1493 & 0.1771 \\
\hline Pt-N33 & 0.0691 & 0.0812 & 0.0580 & 0.0793 & 0.0631 & 0.0768 & 0.0548 & 0.0752 & 0.0467 & 0.0673 & 0.0520 & 0.0843 & 0.1065 & 0.0825 \\
\hline $\mathrm{Pt}-\mathrm{C} 1$ & 0.0832 & 0.0784 & 0.0846 & 0.0799 & 0.0775 & 0.0736 & 0.0833 & 0.0761 & 0.0650 & 0.0606 & 0.0903 & 0.0848 & 0.0727 & 0.0600 \\
\hline Pt-C6 & 0.1054 & 0.1202 & 0.1069 & 0.1183 & 0.1027 & 0.1200 & 0.1019 & 0.1148 & 0.0951 & 0.1113 & 0.1109 & 0.1220 & 0.1088 & 0.1393 \\
\hline \multicolumn{15}{|c|}{ Complex 3} \\
\hline Pt-N27 & 0.1002 & 0.0780 & 0.0880 & 0.0747 & 0.0925 & 0.0734 & 0.0876 & 0.0710 & 0.0850 & 0.0604 & 0.0827 & 0.0781 & 0.1279 & 0.0863 \\
\hline Pt-N28 & 0.1001 & 0.1101 & 0.0880 & 0.1057 & 0.0926 & 0.1093 & 0.0876 & 0.0993 & 0.0847 & 0.1058 & 0.0828 & 0.1055 & 0.1277 & 0.1479 \\
\hline $\mathrm{Pt}-\mathrm{C} 1$ & 0.0850 & 0.1028 & 0.0865 & 0.1021 & 0.0819 & 0.1021 & 0.0841 & 0.0987 & 0.0724 & 0.0956 & 0.0875 & 0.1035 & 0.0833 & 0.1137 \\
\hline Pt-C10 & 0.0850 & 0.0869 & 0.0865 & 0.0880 & 0.0819 & 0.0824 & 0.0841 & 0.0843 & 0.0725 & 0.0703 & 0.0874 & 0.0924 & 0.0834 & 0.0740 \\
\hline \multicolumn{15}{|c|}{ Complex 4} \\
\hline Pt-N33 & 0.1539 & 0.1460 & 0.1387 & 0.1402 & 0.1526 & 0.1431 & 0.1406 & 0.1328 & 0.1162 & 0.1253 & 0.1438 & 0.1429 & 0.1570 & 0.1763 \\
\hline Pt-N34 & 0.0519 & 0.0360 & 0.0426 & 0.0346 & 0.0446 & 0.0326 & 0.0447 & 0.0325 & 0.0405 & 0.0341 & 0.0386 & 0.0391 & 0.0828 & 0.0353 \\
\hline $\mathrm{Pt}-\mathrm{C} 1$ & 0.0857 & 0.0815 & 0.0864 & 0.0838 & 0.0756 & 0.0749 & 0.0847 & 0.0806 & 0.0633 & 0.0571 & 0.0926 & 0.0900 & 0.0613 & 0.0467 \\
\hline Pt-C6 & 0.1081 & 0.1203 & 0.1088 & 0.1183 & 0.1085 & 0.1195 & 0.1030 & 0.1151 & 0.0964 & 0.1093 & 0.1125 & 0.1225 & 0.1162 & 0.1375 \\
\hline \multicolumn{15}{|c|}{ Complex 5} \\
\hline Pt-N27 & 0.1523 & 0.1520 & 0.1351 & 0.1459 & 0.1372 & 0.1501 & 0.1420 & 0.1392 & 0.1128 & 0.1302 & 0.1342 & 0.0224 & 0.1714 & 0.1871 \\
\hline Pt-N28 & 0.0521 & 0.0165 & 0.0449 & & 0.0433 & 0.0072 & & 0.0087 & 0.0442 & 0.0154 & 0.0439 & 0.1473 & 0.0857 & 0.0189 \\
\hline $\mathrm{Pt}-\mathrm{C} 1$ & 0.0840 & 0.0803 & 0.0845 & 0.0829 & 0.0748 & 0.0742 & 0.0829 & 0.0801 & 0.0573 & 0.0566 & 0.0895 & 0.0891 & 0.0509 & 0.0429 \\
\hline Pt-C6 & 0.1080 & 0.1183 & 0.1089 & 0.1163 & 0.1050 & 0.1172 & 0.1033 & 0.1131 & 0.0936 & 0.1077 & 0.1122 & 0.1215 & 0.1186 & 0.1355 \\
\hline \multicolumn{15}{|c|}{ Complex 6} \\
\hline Pt-N34 & 0.1485 & 0.1575 & 0.1353 & 0.1515 & 0.1453 & 0.1560 & 0.1381 & 0.1447 & 0.1161 & 0.1366 & 0.1339 & 0.1521 & 0.1732 & 0.1928 \\
\hline Pt-N35 & 0.0496 & 0.0316 & 0.0396 & 0.0300 & 0.0465 & 0.0268 & 0.0398 & 0.0269 & 0.0378 & 0.0256 & 0.0374 & 0.0356 & 0.0796 & 0.0246 \\
\hline $\mathrm{Pt}-\mathrm{C} 1$ & 0.0834 & 0.0803 & 0.0844 & 0.0827 & 0.0732 & 0.0745 & 0.0829 & 0.0798 & 0.0573 & 0.0578 & 0.0894 & 0.0887 & 0.0503 & 0.0464 \\
\hline Pt-C6 & 0.1070 & 0.1188 & 0.1081 & 0.1167 & 0.1064 & 0.1174 & 0.1028 & 0.1133 & 0.0941 & 0.1066 & 0.1111 & 0.1221 & 0.1187 & 0.1341 \\
\hline
\end{tabular}


TABLE-9

NET NATURAL CHARGES AT THE ACETYL GROUPS (Ac) $)^{\mathrm{a}}$, LIGAND MOIETY AND Pt ${ }^{2+}$ ION OF THE STUDIED COMPLEXES CALCULATED AT THE EXPERIMENTAL AND CALCULATED GEOMETRIES

\begin{tabular}{|c|c|c|c|c|c|c|c|c|c|c|c|c|}
\hline & \multicolumn{2}{|c|}{ B3PW91 } & \multicolumn{2}{|c|}{ M06HF } & \multicolumn{2}{|c|}{ MP2 } & \multicolumn{2}{|c|}{ B3PW91 } & \multicolumn{2}{|c|}{ M06HF } & \multicolumn{2}{|c|}{ MP2 } \\
\hline & Calc. & Exp. & Calc. & Exp. & Calc. & Exp. & Calc. & Exp. & Calc. & Exp. & Calc. & Exp. \\
\hline & \multicolumn{6}{|c|}{ Complex 1} & \multicolumn{6}{|c|}{ Complex 2} \\
\hline $\operatorname{Ac}(1)$ & -0.2418 & -0.2473 & -0.2565 & -0.2582 & -0.3264 & -0.3274 & -0.2431 & -0.2668 & -0.2521 & -0.2752 & -0.3278 & -0.3406 \\
\hline $\operatorname{Ac}(2)$ & -0.2261 & -0.2497 & -0.2501 & -0.2654 & -0.3250 & -0.3359 & -0.2217 & -0.2248 & -0.2444 & -0.2418 & -0.3215 & -0.3138 \\
\hline Sum Ac & -0.4679 & -0.497 & -0.5066 & -0.5236 & -0.6514 & -0.6633 & -0.4648 & -0.4916 & -0.4965 & -0.517 & -0.6493 & -0.6544 \\
\hline $\mathrm{L}$ & 0.2855 & 0.3157 & 0.3204 & 0.3597 & 0.2907 & 0.3303 & 0.2828 & 0.3027 & 0.3140 & 0.3481 & 0.2873 & 0.3184 \\
\hline \multirow[t]{2}{*}{$\mathrm{Pt}$} & 0.1824 & 0.1812 & 0.1862 & 0.1639 & 0.3606 & 0.3329 & 0.1820 & 0.1889 & 0.1825 & 0.1688 & 0.3620 & 0.3359 \\
\hline & \multicolumn{6}{|c|}{ Complex 3} & \multicolumn{6}{|c|}{ Complex 4} \\
\hline $\operatorname{Ac}(1)$ & -0.2418 & -0.2521 & -0.2528 & -0.2624 & -0.3274 & -0.3308 & -0.2447 & -0.2574 & -0.2499 & -0.2661 & -0.3261 & -0.3325 \\
\hline $\operatorname{Ac}(2)$ & -0.2418 & -0.2467 & -0.2528 & -0.2575 & -0.3274 & -0.3263 & -0.2139 & -0.2169 & -0.2424 & -0.2342 & -0.3139 & -0.3054 \\
\hline Sum Ac & -0.4836 & -0.4988 & -0.5056 & -0.5199 & -0.6548 & -0.6571 & -0.4586 & -0.4743 & -0.4923 & -0.5003 & -0.6400 & -0.6379 \\
\hline $\mathrm{L}$ & 0.3031 & 0.3273 & 0.3209 & 0.3586 & 0.2952 & 0.3264 & 0.2734 & 0.2947 & 0.3059 & 0.3331 & 0.2725 & 0.3036 \\
\hline \multirow[t]{2}{*}{$\mathrm{Pt}$} & 0.1805 & 0.1716 & 0.1848 & 0.1614 & 0.3596 & 0.3307 & 0.1852 & 0.1796 & 0.1864 & 0.1671 & 0.3674 & 0.3343 \\
\hline & \multicolumn{6}{|c|}{ Complex 5} & \multicolumn{6}{|c|}{ Complex 6} \\
\hline $\operatorname{Ac}(1)$ & -0.2402 & -0.2739 & -0.2401 & -0.2836 & -0.3242 & -0.3484 & -0.2491 & -0.2766 & -0.2455 & -0.2847 & -0.3275 & -0.3494 \\
\hline $\operatorname{Ac}(2)$ & -0.2127 & -0.2219 & -0.2380 & -0.2406 & -0.3105 & -0.3122 & -0.2077 & -0.2139 & -0.2325 & -0.2308 & -0.3073 & -0.3031 \\
\hline Sum Ac & -0.4529 & -0.4958 & -0.4781 & -0.5242 & -0.6347 & -0.6606 & -0.4568 & -0.4905 & -0.478 & -0.5155 & -0.6348 & -0.6525 \\
\hline L & 0.2673 & 0.2950 & 0.2820 & 0.3354 & 0.2687 & 0.3076 & 0.2661 & 0.2962 & 0.2784 & 0.3377 & 0.2662 & 0.3103 \\
\hline $\mathrm{Pt}$ & 0.1857 & 0.2009 & 0.1961 & 0.1888 & 0.3660 & 0.3530 & 0.1907 & 0.1943 & 0.1996 & 0.1778 & 0.3686 & 0.3422 \\
\hline
\end{tabular}

${ }^{\mathrm{a}} \mathrm{Ac}(1)$ for $\mathrm{CH}_{3}-\mathrm{C}_{(1)}=\mathrm{O}_{(30)}$ and $\mathrm{Ac}(2)$ for $\mathrm{CH}_{3}-\mathrm{C}_{(6)}=\mathrm{O}_{(31)}$

the methods, except MP2, predict very similar net charges for these groups at the experimental and calculated structures. Notably, the MP2 method overestimates the net charge at the Pt-ion compared with the other methods. However, all the methods predict negative charge transfer from the acetyl groups and ligand $(\mathrm{L})$ to the $\mathrm{Pt}^{2+}$ ion. As a result, the charge of the $\mathrm{Pt}^{2+}$ ion decreases to 0.3606 e (exp. 0.3329 e), 0.3620 e (exp. 0.3359 e), 0.3596 e (0.3307 e), 0.3674 e (exp. 0.3343 e), 0.3660 e (exp. 0.3530 e), and 0.3686 e (exp. 0.3422 e) for complexes 1-6, respectively, using the MP2 method.

NBO analysis: NBO analysis is also a significant tool for studying the bonding between atoms in molecular systems by utilizing intramolecular charge transfer (ICT) interactions among NBOs. The stabilization energy $\left(\mathrm{E}^{(2)}\right)$ owing to ICT from an occupied $\mathrm{NBO}\left(\mathrm{NBO}_{\mathrm{i}}\right)$ to an unoccupied one $\left(\mathrm{NBO}_{\mathrm{j}}\right)$ is an important parameter that measures the extent of the interaction among the atoms forming the bond. A high stabilization energy $\left(\mathrm{E}^{(2)}\right)$ indicates a greater donating tendency from the electron donor $\left(\mathrm{NBO}_{\mathrm{i}}\right)$ to the electron acceptor $\left(\mathrm{NBO}_{\mathrm{j}}\right)$. The energies of these interactions were estimated with the aid of second-order perturbation theory [30]. The interaction energies $\left(\mathrm{E}^{(2)}\right)$ obtainedfor the various $\mathrm{Pt}^{2+}$-ligand ICT interactions aresummarized in Table-10.

As can be seen from Table-10, the complexes are stabilized by a set of interesting interactions between the metal ion and ligand NBOs. The ICT interactions among the acetyl groups and $\mathrm{Pt}^{2+}$ NBOs are of two types: (1) from the acetyl ligand occupied NBOs to the $\mathrm{Pt}^{2+}$ antibonding NBOs, which are considered weak as they have low $\mathrm{E}^{(2)}$ values and (2) back donation from the occupied NBOs of $\mathrm{Pt}^{2+}$ to the acetyl group antibonding NBOs, which have high stabilization energies. It is clear that the $\mathrm{Pt}-\mathrm{C}$ bonds are mainly stabilized by $\mathrm{Pt} \rightarrow \mathrm{Ac}$ back donation rather than donation from the ligand to metal ion orbitals, in agreement with the high $\pi$-acidity and strong trans effect of acetyl group. In contrast, the $\mathrm{Pt}-\mathrm{N}$ bonds are stabilized by two types of ICT interactions, both from the ligand moiety to the $\mathrm{Pt}$-ion and $\mathrm{Pt} \rightarrow \mathrm{N}$ back donation is negligible. $\mathrm{L} \rightarrow \mathrm{Pt}$ donation occurs from the lone pair $\mathrm{NBO}$ of the $\mathrm{N}$-atom to either the $\mathrm{Pt}$ or $\mathrm{Pt}-\mathrm{C}$ antibonding NBOs. The strong $\sigma$-donation and negligible back donation of the $\mathrm{N}$-donor hydrazone ligands stabilize the $\mathrm{Pt}-\mathrm{C}$ bonds trans to the corresponding $\mathrm{N}$-atom. As an example, pictorial presentations of these interactions for complex 1 are shown in Figs. 3 and 4. It is clear from Fig. 5 that the acetyl ligand is a weak donor ligand. In contrast, Fig. 4 shows stronger interactions between the filled Pt orbitals and the unoccupied antibonding orbitals of the acetyl ligand. The Pt-N bonds are stabilized only by ICT interactions from the $\mathrm{N}$-donor orbitals as the donor to the Pt antibonding orbitals as the acceptor as shown in Fig. 5.

\section{Conclusion}

Six (B3LYP, CAM-B3LYP, B3PW91, M06, M06HF and PBE) DFT methods and one post-Hartree-Fock (MP2) method were assessed for their accuracy in predicting the $\mathrm{Pt}-\mathrm{C}$ and $\mathrm{Pt}-\mathrm{N}$ bond distances of various diacetyl platinum(II) complexes, $\left[\mathrm{Pt}(\mathrm{Ac})_{2} \mathrm{~L}\right]$. The M06HF and MP2 methods were found to be the best for predicting the $\mathrm{Pt}-\mathrm{N}$ bond distances. The efficiency of the M06HF method is equivalent to that of the computationally expensive MP2 method. For Pt-C bond distances, the MP2 and M06HF methods gave the worst predictions. The other methods, in particular, the B3PW91 method, predicted the $\mathrm{Pt}-\mathrm{C}$ bond distances very well. With all the methods, the AIM topological parameters indicated the Pt-N (pyridine) bonds have higher interaction energies and covalent characters than the Pt-N (hydrazone) bonds. In addition, the $\mathrm{Pt}-\mathrm{C}$ bonds trans to the $\mathrm{Pt}-\mathrm{N}$ (hydrazone) bonds are more likely to be covalent than the $\mathrm{Pt}-\mathrm{C}$ bonds trans to the $\mathrm{Pt}-\mathrm{N}$ (pyridine) bonds. Thus, AIM is a sensitive tool for comparing bonding natures, irrespective of the computational method used. NBO studies predicted negative charge transfer from the ligand moieties (Ac and L) to the $\mathrm{Pt}^{2+}$ ion and the MP2 method over estimated the net charge at the Pt-ion. The results indicated 


\begin{tabular}{|c|c|c|c|c|c|c|}
\hline \multirow{3}{*}{$\mathrm{NBO}_{\mathrm{i}} \rightarrow \mathrm{NBO}_{\mathrm{j}}^{\mathrm{a}}$} & \multicolumn{5}{|c|}{$\begin{array}{l}\text { TABLE-10 } \\
\text { INTRAMOLECULAR CHARGE TRANSFER INTERACTIONS AMONG Pt }{ }^{2+} \text { AND } \\
\text { LIGAND NATURAL BOND ORBITALS DETERMINED USING NBO ANALYSIS }\end{array}$} & \multirow{2}{*}{ MP2 } \\
\hline & \multicolumn{2}{|c|}{ B3PW91 } & \multicolumn{2}{|c|}{ M06HF } & \multirow[b]{2}{*}{ Calc. } & \\
\hline & Calc. & Exp. & Calc. & Exp. & & Exp. \\
\hline \multicolumn{7}{|c|}{ Complex 1} \\
\hline $\mathrm{Ac}(\mathrm{C} 1-\mathrm{O} 30) \rightarrow \mathrm{Pt}$ & 3.00 & 2.17 & 3.07 & 2.01 & 2.97 & 1.74 \\
\hline $\mathrm{Ac}(\mathrm{C} 6-\mathrm{O} 31) \rightarrow \mathrm{Pt}$ & 2.37 & 2.23 & 2.95 & 2.04 & 2.55 & 1.76 \\
\hline $\mathrm{LP}(\mathrm{Pt}) \rightarrow \mathrm{Ac}(\mathrm{C} 1-\mathrm{O} 30)$ & 19.21 & 16.83 & 24.04 & 18.34 & 22.12 & 16.66 \\
\hline $\mathrm{LP}(\mathrm{Pt}) \rightarrow \mathrm{Ac}(\mathrm{C} 6-\mathrm{O} 31)$ & 17.73 & 17.36 & 22.56 & 19.26 & 20.26 & 17.70 \\
\hline $\mathrm{LP}(\mathrm{N} 25) \rightarrow \mathrm{Pt}$ & 38.21 & 44.49 & 57.64 & 65.46 & 61.15 & 67.44 \\
\hline $\mathrm{LP}(\mathrm{N} 26) \rightarrow \mathrm{Pt}$ & 38.53 & 44.25 & 56.74 & 65.43 & 60.17 & 67.00 \\
\hline $\mathrm{LP}(\mathrm{N} 25) \rightarrow \mathrm{BD}^{*}(1) \mathrm{C} 6-\mathrm{Pt}$ & 36.87 & 45.18 & 48.20 & 54.28 & 45.99 & 51.48 \\
\hline $\mathrm{LP}(\mathrm{N} 26) \rightarrow \mathrm{BD}^{*}(1) \mathrm{C} 1-\mathrm{Pt}$ & 40.50 & 46.24 & 50.05 & 55.41 & 47.40 & 52.00 \\
\hline \multicolumn{7}{|c|}{ Complex 2} \\
\hline $\mathrm{Ac}(\mathrm{C} 1-\mathrm{O} 37) \rightarrow \mathrm{Pt}$ & 2.99 & 3.22 & 3.14 & 3.04 & 2.92 & 2.86 \\
\hline $\mathrm{Ac}(\mathrm{C} 6-\mathrm{O} 38) \rightarrow \mathrm{Pt}$ & 2.38 & 3.18 & 2.49 & 2.96 & 4.69 & 2.73 \\
\hline $\mathrm{LP}(\mathrm{Pt}) \rightarrow \mathrm{Ac}(\mathrm{C} 1-\mathrm{O} 37)$ & 19.20 & 19.04 & 23.42 & 19.93 & 22.01 & 18.46 \\
\hline $\mathrm{LP}(\mathrm{Pt}) \rightarrow \mathrm{Ac}(\mathrm{C} 6-\mathrm{O} 38)$ & 15.41 & 12.77 & 19.03 & 14.96 & 17.68 & 13.51 \\
\hline $\mathrm{LP}(\mathrm{N} 32) \rightarrow \mathrm{Pt}$ & 36.92 & 42.74 & 54.95 & 62.11 & 59.50 & 63.23 \\
\hline $\mathrm{LP}(\mathrm{N} 33) \rightarrow \mathrm{Pt}$ & 38.47 & 44.56 & 57.82 & 65.73 & 60.24 & 67.47 \\
\hline $\mathrm{LP}(\mathrm{N} 32) \rightarrow \mathrm{BD}^{*}(1) \mathrm{C} 6-\mathrm{Pt}$ & 36.10 & 41.97 & 45.72 & 50.88 & 45.57 & 48.28 \\
\hline $\mathrm{LP}(\mathrm{N} 33) \rightarrow \mathrm{BD}^{*}(1) \mathrm{C} 1-\mathrm{Pt}$ & 40.80 & 49.18 & 51.48 & 58.63 & 47.82 & 55.12 \\
\hline \multicolumn{7}{|c|}{ Complex 3} \\
\hline $\mathrm{Ac}(\mathrm{C} 1-\mathrm{O} 29) \rightarrow \mathrm{Pt}$ & 0.63 & 0.00 & 0.84 & 0.00 & 0.85 & 0 \\
\hline $\mathrm{Ac}(\mathrm{C} 10-\mathrm{O} 30) \rightarrow \mathrm{Pt}$ & 0.63 & 0.70 & 0.84 & 0.75 & 0.85 & 0.85 \\
\hline $\mathrm{Pt} \rightarrow \mathrm{Ac}(\mathrm{C} 1-\mathrm{O} 29)$ & 19.45 & 17.62 & 24.20 & 11.60 & 21.64 & 17.47 \\
\hline $\mathrm{Pt} \rightarrow \mathrm{Ac}(\mathrm{C} 10-\mathrm{O} 30)$ & 19.45 & 17.68 & 24.22 & 26.00 & 21.66 & 17.53 \\
\hline $\mathrm{LP}(\mathrm{N} 27) \rightarrow \mathrm{Pt}$ & - & - & - & - & - & - \\
\hline $\mathrm{LP}(\mathrm{N} 28) \rightarrow \mathrm{Pt}$ & - & - & - & - & - & - \\
\hline $\mathrm{LP}(\mathrm{N} 27) \rightarrow \mathrm{BD}^{*}(1) \mathrm{C} 10-\mathrm{Pt}$ & - & - & - & - & - & - \\
\hline $\mathrm{LP}(\mathrm{N} 28) \rightarrow \mathrm{BD}^{*}(1) \mathrm{C} 1-\mathrm{Pt}$ & - & - & - & - & - & - \\
\hline \multicolumn{7}{|c|}{ Complex 4} \\
\hline $\mathrm{Ac}(\mathrm{C} 1-\mathrm{O} 37) \rightarrow \mathrm{Pt}$ & 3.01 & 3.17 & 3.17 & 3.03 & 2.96 & 2.88 \\
\hline $\mathrm{Ac}(\mathrm{C} 6-\mathrm{O} 38) \rightarrow \mathrm{Pt}$ & 2.50 & 3.29 & 2.57 & 3.13 & 2.15 & 2.82 \\
\hline $\mathrm{Pt} \rightarrow \mathrm{Ac}(\mathrm{C} 1-\mathrm{O} 37)$ & 20.02 & 18.78 & 24.83 & 17.07 & 22.88 & 18.55 \\
\hline $\mathrm{Pt} \rightarrow \mathrm{Ac}(\mathrm{C} 6-\mathrm{O} 38)$ & 17.66 & 16.21 & 22.52 & 21.31 & 19.86 & 16.01 \\
\hline $\mathrm{LP}(\mathrm{N} 33) \rightarrow \mathrm{Pt}$ & 34.13 & 43.05 & 54.50 & 62.59 & 56.49 & 63.09 \\
\hline $\mathrm{LP}(\mathrm{N} 34) \rightarrow \mathrm{Pt}$ & 38.05 & 43.45 & 57.93 & 64.71 & 61.12 & 66.55 \\
\hline $\mathrm{LP}(\mathrm{N} 33) \rightarrow \mathrm{BD}^{*}(1) \mathrm{C} 6-\mathrm{Pt}$ & 33.86 & 41.39 & 46.02 & 50.45 & 44.55 & 47.74 \\
\hline $\mathrm{LP}(\mathrm{N} 34) \rightarrow \mathrm{BD}^{*}(1) \mathrm{C} 1-\mathrm{Pt}$ & 41.31 & 48.85 & 51.80 & 57.92 & 48.86 & 54.42 \\
\hline \multicolumn{7}{|c|}{ Complex 5} \\
\hline $\mathrm{Ac}(\mathrm{C} 1-\mathrm{O} 31) \rightarrow \mathrm{Pt}$ & 3.07 & 3.53 & 3.14 & 3.39 & 3.02 & 3.65 \\
\hline $\mathrm{Ac}(\mathrm{C} 6-\mathrm{O} 32) \rightarrow \mathrm{Pt}$ & 2.47 & 3.49 & 2.56 & 3.26 & 2.30 & 3.66 \\
\hline $\mathrm{Pt} \rightarrow \mathrm{Ac}(\mathrm{C} 1-\mathrm{O} 31)$ & 19.89 & 19.6 & 26.12 & 21.83 & 22.89 & 17.99 \\
\hline $\mathrm{Pt} \rightarrow \mathrm{Ac}(\mathrm{C} 6-\mathrm{O} 32)$ & 17.45 & 16.97 & 22.16 & 18.96 & 20.21 & 15.65 \\
\hline $\mathrm{LP}(\mathrm{N} 27) \rightarrow \mathrm{Pt}$ & 35.93 & 42.84 & 56.31 & 62.28 & 57.82 & 38.01 \\
\hline $\mathrm{LP}(\mathrm{N} 28) \rightarrow \mathrm{Pt}$ & 37.58 & 42.30 & 58.58 & 63.12 & 60.62 & 37.01 \\
\hline $\mathrm{LP}(\mathrm{N} 27) \rightarrow \mathrm{BD}^{*}(1) \mathrm{C} 6-\mathrm{Pt}$ & 34.28 & 40.62 & 46.05 & 49.78 & 43.37 & 37.60 \\
\hline $\mathrm{LP}(\mathrm{N} 28) \rightarrow \mathrm{BD}^{*}(1) \mathrm{C} 1-\mathrm{Pt}$ & 39.95 & 47.46 & 50.26 & 56.58 & 47.57 & 44.17 \\
\hline \multicolumn{7}{|c|}{ Complex 6} \\
\hline $\mathrm{Ac}(\mathrm{C} 1-\mathrm{O} 38) \rightarrow \mathrm{Pt}$ & 3.04 & 3.46 & 3.09 & 3.59 & 3.03 & 3.31 \\
\hline $\mathrm{Ac}(\mathrm{C} 6-\mathrm{O} 39) \rightarrow \mathrm{Pt}$ & 2.48 & 3.45 & 2.63 & 3.61 & 2.23 & 3.22 \\
\hline $\mathrm{Pt} \rightarrow \mathrm{Ac}(\mathrm{C} 1-\mathrm{O} 38)$ & 20.43 & 19.94 & 26.36 & 18.28 & 23.65 & 21.83 \\
\hline $\mathrm{Pt} \rightarrow \mathrm{Ac}(\mathrm{C} 6-\mathrm{O} 39)$ & 17.57 & 16.51 & 22.02 & 15.12 & 19.64 & 18.79 \\
\hline $\mathrm{LP}(\mathrm{N} 34) \rightarrow \mathrm{Pt}$ & 35.34 & 42.66 & 55.90 & 37.88 & 57.33 & 61.77 \\
\hline $\mathrm{LP}(\mathrm{N} 35) \rightarrow \mathrm{Pt}$ & 37.75 & 42.61 & 59.27 & 37.27 & 60.59 & 63.41 \\
\hline $\mathrm{LP}(\mathrm{N} 34) \rightarrow \mathrm{BD}^{*}(1) \mathrm{C} 6-\mathrm{Pt}$ & 33.36 & 40.23 & 45.40 & 37.31 & 42.88 & 49.27 \\
\hline $\mathrm{LP}(\mathrm{N} 35) \rightarrow \mathrm{BD}^{*}(1) \mathrm{C} 1-\mathrm{Pt}$ & 40.69 & 48.55 & 51.72 & 45.27 & 48.11 & 57.84 \\
\hline
\end{tabular}



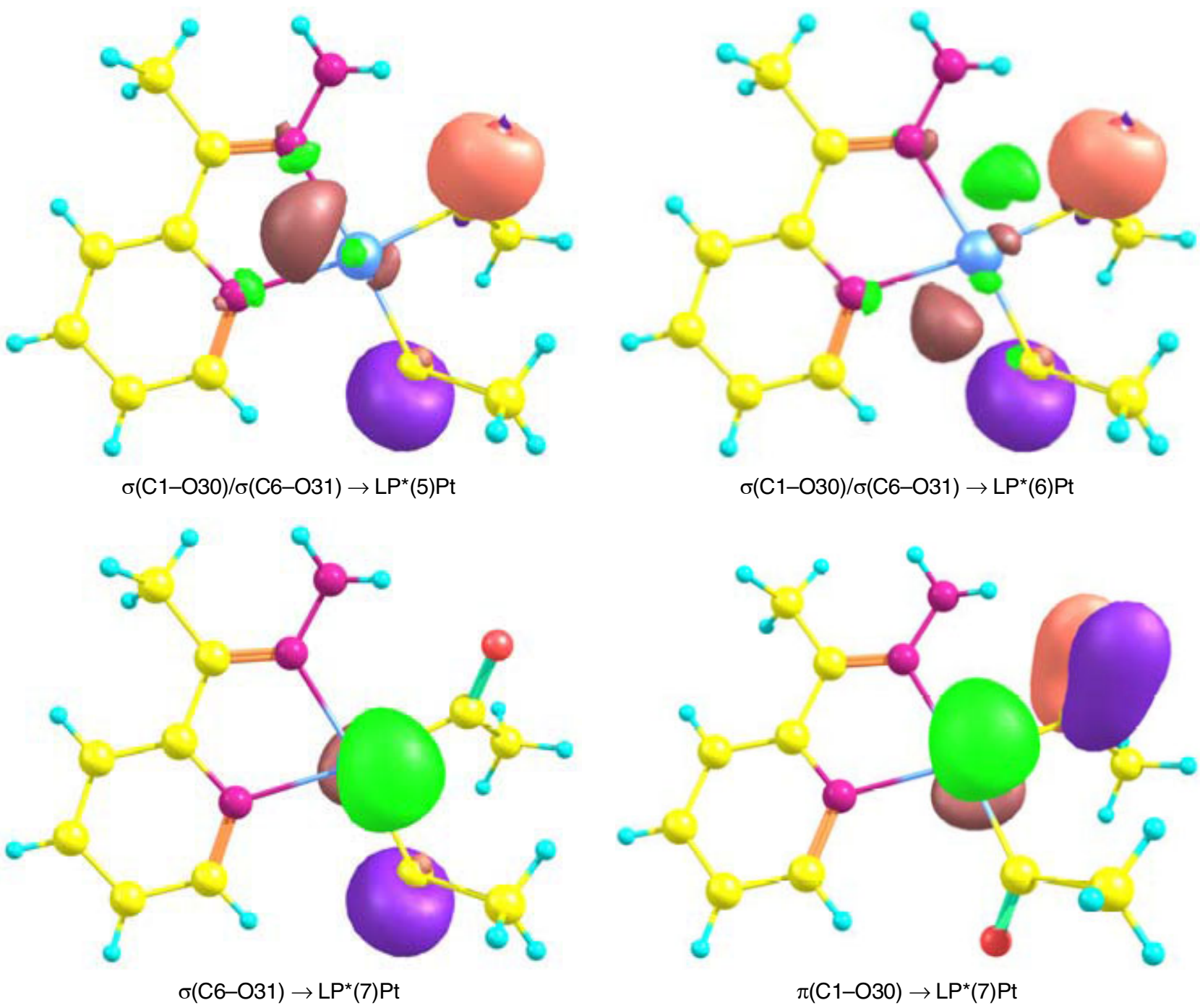

Fig. 3. Insignificant interactions between the filled natural orbitals of acetyl groups and unoccupied Pt-orbitals, as obtained using the MP2 method; Pt orbitals are green and brown and acetyl orbitals are orange and purple

that $\mathrm{Ac} \rightarrow \mathrm{Pt} \sigma$-bonding is not important, as the $\mathrm{Pt}-\mathrm{C}$ bonds are mainly stabilized by $\mathrm{Pt} \rightarrow \mathrm{Ac}$ back donation, in agreement with the high $\pi$-acidity and strong trans effect of acetyl groups. In contrast, the Pt-N bonds are stabilized only by $\mathrm{N} \rightarrow \mathrm{Pt} \sigma$ donation, which stabilizes the trans $\mathrm{Pt}-\mathrm{C}$ bonds.

\section{ACKNOWLEDGEMENTS}

The authors extend their appreciation to the Deanship of Scientific Research at King Saud University for funding the work throughresearch group no. RGP-038.

\section{CONFLICT OF INTEREST}

The authors declare that there is no conflict of interests regarding the publication of this article.

\section{REFERENCES}

1. B. Rosenberg, L. Vancamp, J.E. Trosko and V.H. Mansour, Nature, 222 , 385 (1969);

https://doi.org/10.1038/222385a0.

2. P.J. O'Dwyer, J.P. Stevenson and S.W. Johnson, ed.: B. Lippert, In: Cisplatin, Chemistry and Biochemistry of a Leading Anticancer Drug, WileyVCH: Weinheim, Germany (1999).
3. M. Watson, A. Barret, R. Spence and C. Twelves, Oncology, Oxford University Press: Oxford, edn 2 (2006).

4. C.A. Rabik and M.E. Dolan, Cancer Treat. Rev., 33, 9 (2007); https://doi.org/10.1016/j.ctrv.2006.09.006.

5. M. Galanski, M. Jakupec and B. Keppler, Curr. Med. Chem., 12, 2075 (2005); https://doi.org/10.2174/0929867054637626.

6. D. Wang and S.J. Lippard, Nat. Rev. Drug Discov., 4, 307 (2005); https://doi.org/10.1038/nrd1691.

7. N.J. Wheate, S. Walker, G.E. Craig and R. Oun, Dalton Trans., 39, 8113 (2010); https://doi.org/10.1039/c0dt00292e.

8. S. Dhar and S.J. Lippard, ed.: E. Alessp, Bioinorganic Medicinal Chemistry, Wiley-VCH: Weinheim, Germany, pp. 79-95 (2011).

9. X. Wang and Z. Guo, ed.: E. Alessp, Bioinorganic Medicinal Chemistry, Wiley-VCH: Weinheim, Germany, pp. 97-149 (2011).

10. P. Štarha, Z. Trávníèek, A. Popa, I. Popa, T. Muchová and V. Brabec, J. Inorg. Biochem., 115, 57 (2012); https://doi.org/10.1016/j.jinorgbio.2012.05.006.

11. N. Ferri, S. Cazzaniga, L. Mazzarella, G. Curigliano, G. Lucchini, D. Zerla, R. Gandolfi, G. Facchetti, M. Pellizzoni and I. Rimoldi, Bioorg. Med. Chem., 21, 2379 (2013); https://doi.org/10.1016/j.bmc.2013.01.063.

12. C. Icsel, V.T. Yilmaz, F. Ari, E. Ulukaya and W.T.A. Harrison, Eur. J. Med. Chem., 60, 386 (2013); https://doi.org/10.1016/j.ejmech.2012.12.002. 


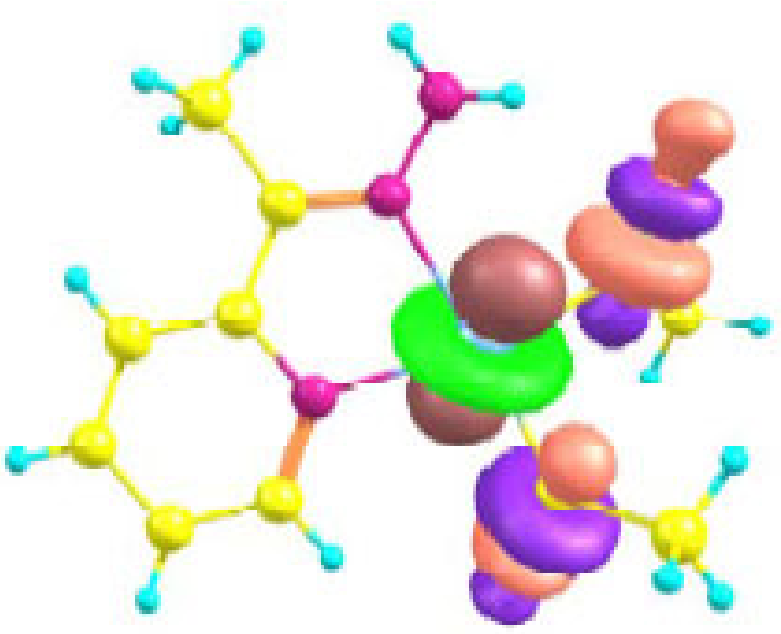

$\mathrm{LP}(2) \mathrm{Pt} \rightarrow \sigma^{\star}(\mathrm{C} 1-\mathrm{O} 30) / \sigma^{\star}(\mathrm{C} 6-\mathrm{O} 31)$

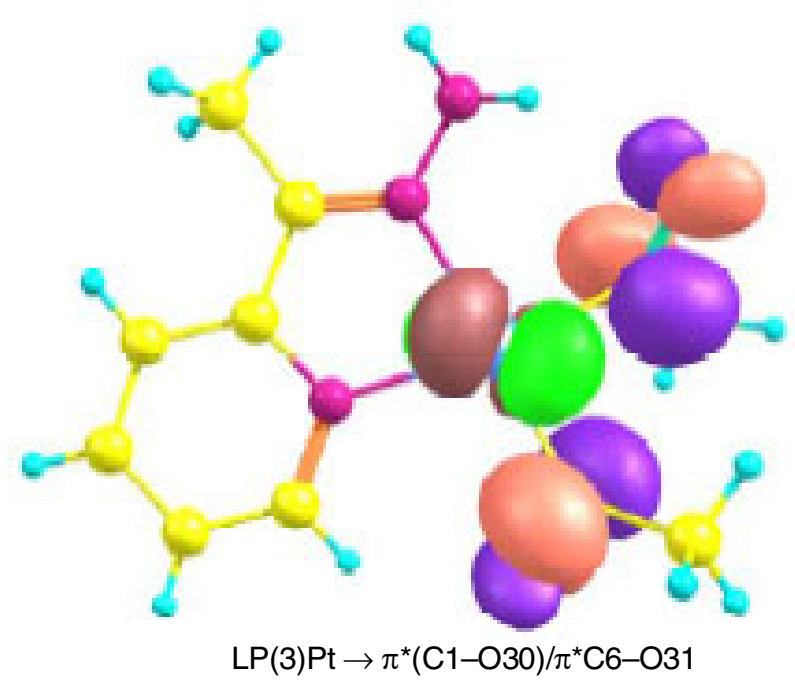

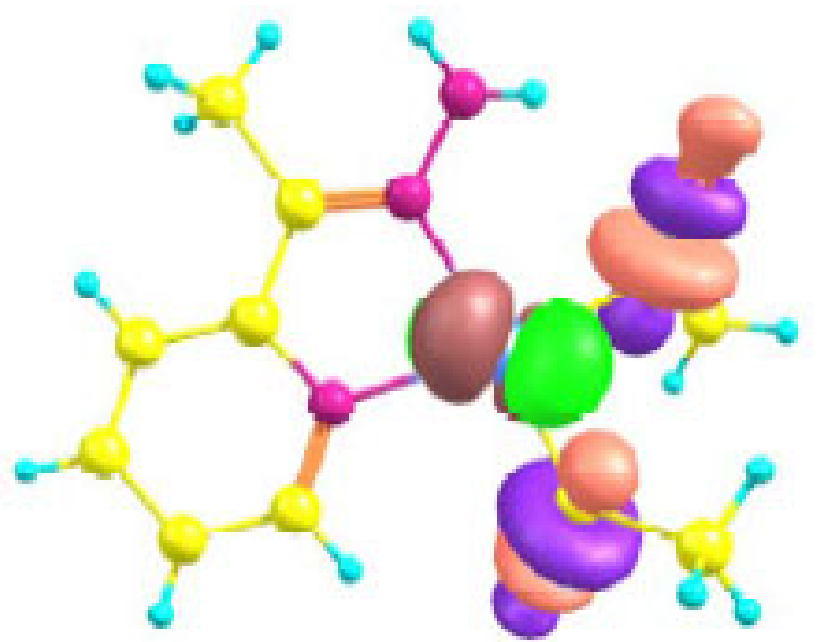

$\mathrm{LP}(3) \mathrm{Pt} \rightarrow \sigma^{\star}(\mathrm{C} 1-\mathrm{O} 30) / \sigma^{\star}(\mathrm{C} 6-\mathrm{O} 31)$

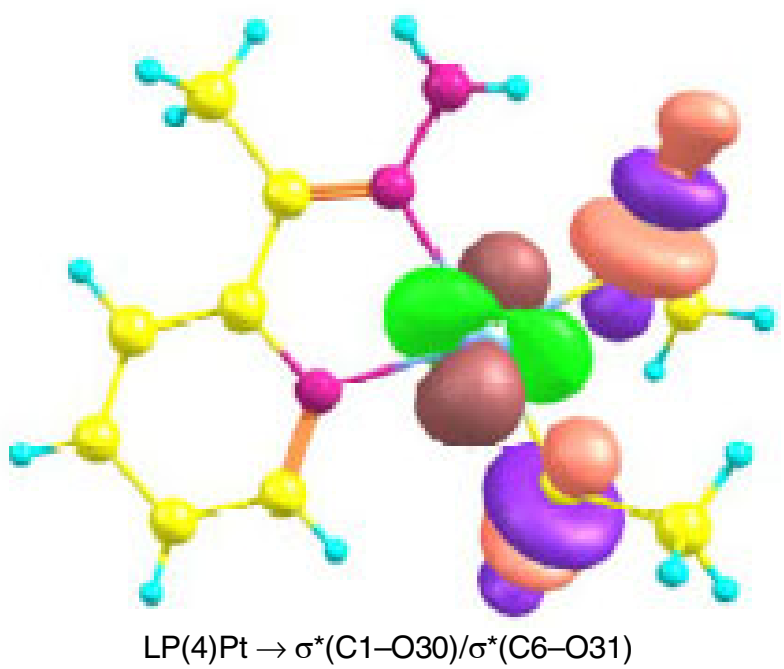

Fig. 4. Interactions owing to metal $\pi$-back donation between the filled natural orbitals of the Pt-ion and the unoccupied Ac orbitals, which are stronger than those owing to Ac $\rightarrow$ Pt donation, as obtained using the MP2 method; Pt orbitals are green and brown and Ac orbitals are orange and purple

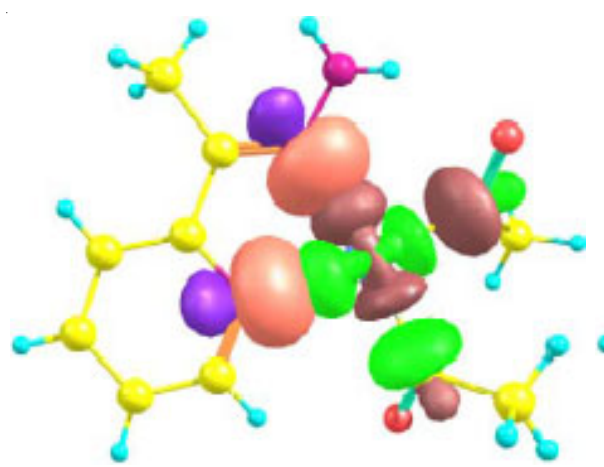

$\operatorname{LPN}(25) / \operatorname{LPN}(26) \rightarrow \sigma(\mathrm{C} 6-\mathrm{Pt}) / \sigma(\mathrm{C} 1-\mathrm{Pt})$

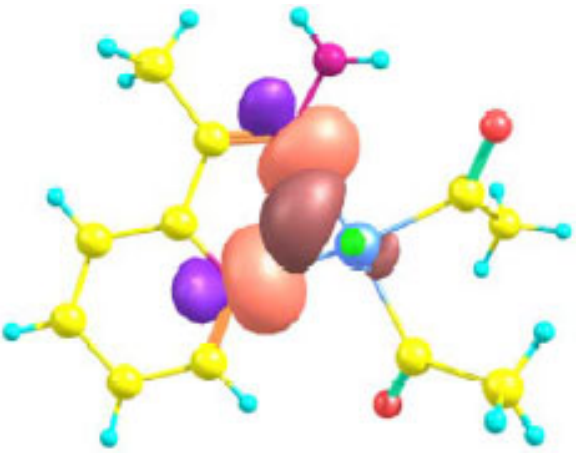

$\operatorname{LPN}(25) / L P N(26) \rightarrow \operatorname{LP}^{*}(5) P t$

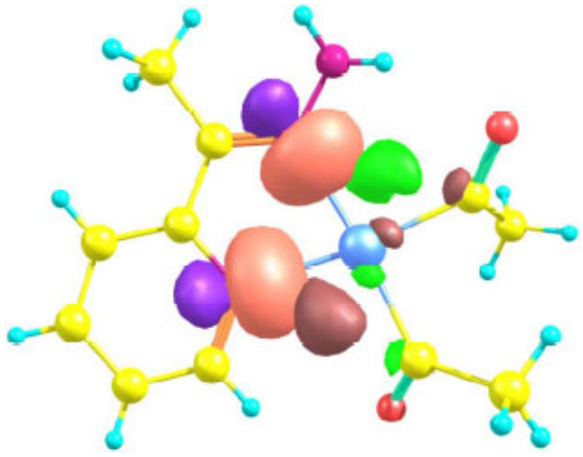

$\operatorname{LPN}(25) / \mathrm{LPN}(26) \rightarrow \mathrm{LP}^{*}(6) \mathrm{Pt}$

Fig. 5. Strong interactions owing to $\sigma$-donation from the $N$ lone pair (LP) orbitals of the hydrazone ligand to the unoccupied metal orbitals, as obtained using the MP2 method; Pt orbitals are green and brown, and the LP orbitals of nitrogen are orange and purple

13. R.C. Johnstone, G.Y. Park and S.J. Lippard, Anticancer Res., 34, 471 (2014).

14. P.L.A. Popelier and G.J. Logothetis, J. Organomet. Chem., 555, 101 (1998); https://doi.org/10.1016/S0022-328X(97)00710-9.

15. R.F.W. Bader, Atoms in Molecules: A Quantum Theory, Clarendon Press Oxford (1990).

16. R.F.W. Bader and H. Essen, J. Chem. Phys., 80, 1943 (1984); https://doi.org/10.1063/1.446956.
17. T. Kluge, E. Bette, M. Bette, J. Schmidt and D. Steinborn, J. Organomet. Chem., 762, 48 (2014); https://doi.org/10.1016/j.jorganchem.2014.03.030.

18. M.J. Frisch, G.W. Trucks, H.B. Schlegel, G.E. Scuseria, M.A. Robb, J.R. Cheeseman, G. Scalmani, V. Barone, B. Mennucci, G.A. Petersson, H. Nakatsuji, M. Caricato, X. Li, H.P. Hratchian, A.F. Izmaylov, J. Bloino, G. Zheng, J.L. Sonnenberg, M. Hada, M. Ehara, K. Toyota, R. 
Fukuda, J. Hasegawa, M. Ishida, T. Nakajima, Y. Honda, O. Kitao, H. Nakai, T. Vreven, J.A. Montgomery Jr., J.E. Peralta, F. Ogliaro, M. Bearpark, J.J. Heyd, E. Brothers, K.N. Kudin, V.N. Staroverov, R. Kobayashi, J. Normand, K. Raghavachari, A. Rendell, J.C. Burant, S.S. Iyengar, J. Tomasi, M. Cossi, N. Rega, J.M. Millam, M. Klene, J.E. Knox, J.B. Cross, V. Bakken, C. Adamo, J. Jaramillo, R. Gomperts, R.E. Stratmann, O. Yazyev, A.J. Austin, R. Cammi, C. Pomelli, J.W. Ochterski, R.L. Martin, K. Morokuma, V.G. Zakrzewski, G.A. Voth, P. Salvador, J.J. Dannenberg, S. Dapprich, A.D. Daniels, O. Farkas, J.B. Foresman, J.V. Ortiz, J. Cioslowski and D.J. Fox, Gaussian Inc., Wallingford CT (2009).

19. A.D. Becke, J. Chem. Phys., 98, 5648 (1993); https://doi.org/10.1063/1.464913.

20. C. Lee, W. Yang and R.G. Parr, Phys. Rev. B, 37, 785 (1988); https://doi.org/10.1103/PhysRevB.37.785.

21. T. Yanai, D. Tew and N. Handy, Chem. Phys. Lett., 393, 51 (2004); https://doi.org/10.1016/j.cplett.2004.06.011.

22. X. Xu and W.A. Goddard III, Proc. Natl. Acad. Sci. USA, 101, 2673 (2004); https://doi.org/10.1073/pnas.0308730100.

23. Y. Zhao and D.G. Truhlar, Theor. Chem. Acc., 120, 215 (2008); https://doi.org/10.1007/s00214-007-0310-x.

24. J.P. Perdew, K. Burke and M. Ernzerhof, Phys. Rev. Lett., 78, 1396 (1997);

https://doi.org/10.1103/PhysRevLett.78.1396.
25. J.P. Blaudeau, M.P. McGrath, L.A. Curtiss and L. Radom, J. Chem. Phys., 107, 5016 (1997); https://doi.org/10.1063/1.474865.

26. P.J. Hay and W.R. Wadt, J. Chem. Phys., 82, 270 (1985); https://doi.org/10.1063/1.448799.

27. A. Üngördü and N. Tezer, J. Saudi Chem. Soc., 21, 837 (2017); https://doi.org/10.1016/j.jscs.2017.04.003.

28. C. Møller and M.S. Plesset, Phys. Rev., 46, 618 (1934); https://doi.org/10.1103/PhysRev.46.618.

29. T. Lu and R. Chen, J. Comput. Chem., 33, 580 (2012); https://doi.org/10.1002/jcc.22885.

30. F. Weinhold and J.E. Carpenter, The Natural Bond Orbital Lewis Structure Concept for Molecules, Radicals and Radical Ions, Plenum, pp. 227 (1988).

31. D. Cremer and D. Kraka, Angew. Chem. Int., 23, 627 (1984); https://doi.org/10.1002/anie.198406271.

32. E. Espinosa, I. Alkorta, J. Elguero and E. Molins, J. Chem. Phys., 117, 5529 (2002); https://doi.org/10.1063/1.1501133.

33. D. Cremer and E. Kraka, Croat. Chem. Acta, 57, 1259 (1984).

34. E. Espinosa, E. Molins and C. Lecomte, Chem. Phys. Lett., 285, 170 (1998); https://doi.org/10.1016/S0009-2614(98)00036-0.

35. C.S. López, O.N. Faza, F.P. Cossío, D.M. York and A.R. de Lera, Chem. Eur. J., 11, 1734 (2005);

https://doi.org/10.1002/chem.200401026. 